\title{
System of Systems Simulation driven Urban Air Mobility Vehicle Design and Fleet Assessment
}

\author{
Prajwal Shiva Prakasha*, Nabih Naeem ${ }^{\dagger}$, Patrick Ratei, Björn Nagel ${ }^{\S}$ \\ German Aerospace Center (DLR), Institute of System Architectures in Aeronautics, Hamburg, 21129, Germany \\ Oliver Bertram** \\ German Aerospace Center (DLR), Institute of Flight Systems, Braunschweig, 38108, Germany
}

\begin{abstract}
Urban Air Mobility (UAM) is increasingly becoming popular for Passenger or Cargo movement in dense smart cities. Several researches so far are focused on individual vehicle architectures such as multirotor or tiltrotor etc., but not much effort in a System of Systems (SoS) point of view where a homogenous fleet of vehicle with different passenger capacity, speed, and propulsive energy concepts are assessed in a framework for a successful UAM operations in a given city. An effort is made in this paper wherein, vehicle architecture is derived from the Concept of Operations (CONOPS) of scenarios such as urban and suburban operations and as well as propulsion subsystem for sustainable UAM. This paper approaches UAM aircraft design driven by SoS approach and an agent-based simulation supports the vehicle architecture evaluation and fleet definition.
\end{abstract}

The outcome of this study is: multiple aircraft design with subsystem architectures, ideal fleet size for the respective operational scenarios, autonomy and battery technology effectiveness on UAM throughput (to efficiently provide UAM on-demand service maximum passengers within 15 min wait time), and importantly, sustainability metrics such as total fleet energy required. Several System of Systems, system and subsystem level sensitivity research questions are addressed to understand the interlevel coupling.

\section{Introduction}

SINCE the release of the Uber Elevate white paper [1] in 2016, the field of Urban Air Mobility (UAM) has gained a

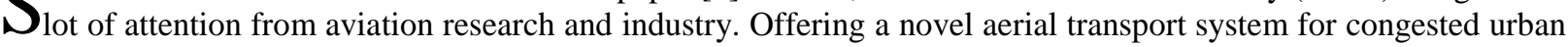
areas, established and startup aircraft manufacturers have proposed various design concepts for the required air taxis or UAM aircraft. By the use of distributed electric propulsion, mostly fully electric Vertical Take-Off and Landing (eVTOL) vehicles or aircraft have been considered for UAM operations. However, there are various eVTOL aircraft configurations or architectures among the presented concepts.

From an aircraft performance-based perspective these architectures can be characterized by their hover and cruise efficiency, i.e. disk loading and lift-to-drag ratio. Estimates of the design space for potential eVTOL aircraft architectures in terms of the aforementioned design variables have been provided by McDonald and German [2] and and are reproduced in Fig. 1. Thus, two major groups, which are distinguished by their lift production in cruise flight, can be identified. While multirotor, conventional, and coaxial helicopters are classified as rotary-wing cruise architectures (see shades of red in Fig. 1), lift + cruise, tilt duct, tilt wing, and tilt rotor aircraft are categorized as fixed-wing cruise architectures (see shades of blue in Fig. 1). The compound helicopter architecture combines both, rotary-wing as well as fixed-wing cruise, to create lift and is therefore situated in-between the two major groups, but is still classified as rotary-wing cruise configuration.

Multirotor vehicles, such as the single-seater EHang 216 [3] or the two-seater VoloCity by Volocopter [4], may be more efficient in hovering flight state due to lower disk loading, but do only fulfil shorter cruise missions. Jaunt Air Mobility is developing the Jaunt Journey Air Taxi [5], a compound helicopter configuration, that should perform

\footnotetext{
${ }^{*}$ Research Scientist, Institute of System Architectures in Aeronautics, AIAA Member.

$\dagger$ Research Student, Institute of System Architectures in Aeronautics.

* Research Student, Institute of System Architectures in Aeronautics.

$\S$ Founding Director and Head of Institute, Institute of System Architectures in Aeronautics.

${ }^{* *}$ Research Group Leader and Deputy Head of Department, Institute of Flight Systems.
} 
similar in hover, but performs more efficient in cruise flight state. Fixed-wing cruise architectures may not be as optimal for hover as the aforementioned configurations, but offer greater ranges due to higher lift-to-drag ratio, i.e. cruise efficiency. Within this group, eVTOL aircraft, such as two-seater lift + cruise Wisk Cora [6], five-seater tilt rotor Joby S4 [7], and five-seater tilt duct Lilium Jet [8], can be found.

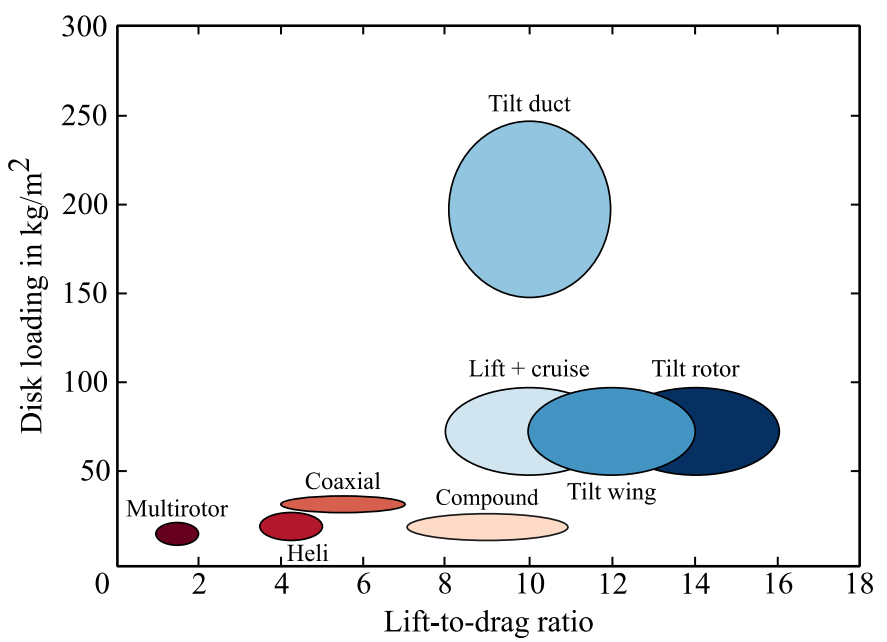

Fig. 1 Conceptual design space for different eVTOL aircraft configurations based on [2]

Due to the aircraft performance requirements, the different types of eVTOL will also have different battery power requirements. These battery power requirements will also vary with the environmental conditions and mission requirements such as cruise speed, payload or reserves. Thus, the battery size and dimensions will change with a strong impact on the overall aircraft size and weight.

Today, electric propulsion and battery technology are the key enablers for the realization of eVTOL concepts. But the today's available battery technology ends in high component weight with reduced payload capabilities and only allows for short flight times or endurances in eVTOL applications. This has a major impact on the aircraft design and operational aspects of the individual eVTOL, the entire eVTOL fleet, the vertiports or, in other words, the overall system of systems. As battery technology parameters such as specific power or energy density improve, however, payload, cruise speeds and distances will increase accordingly. To meet these existing challenges in today's battery technology hybrid-electric powertrain concepts can be used as bridge technology alternatively.

\section{A. Urban Air Mobility as System of Systems}

The term System of Systems (SoS) has been recently formalized by ISO 21839:2019 [9] as a set of systems that interact and provide unique capabilities that the individual constituent systems cannot accomplish on their own. SoS is an emergent concept that is widely accepted as an approach to understand complex systems in fields such as renewable energy, national security, infrastructure, transport, and defense [10]. Although each field has adopted a specialized definition of SoS to suit their needs, the influential work of Maier [11] has provided a widely accepted characterization of SoS [12]. These are referred to as the Maier criteria or OMGEE characteristics [13] and are namely: Operational as well as Managerial independence, Geographic distribution, Emergence, and Evolutionary behavior.

Urban Air Mobility, as shown in Fig. 2, has constituent systems/Systems of Interest such as the vehicle, the heterogeneous fleet, the vertiport system, air traffic operations (trajectory, conflict resolution, safety), the passenger demand, the energy system, its life-cycle aspects at vehicle, fleet and grid level. These systems are operationally and managerially independent. The constituent systems/ system of Interests combined to make a SoS, which needs to collaborate for successful efficient operation or to achieve positive emergence. Moreover, each of the constituent systems is geographically distributed and evolves independently at individual points in time (e.g. new vehicles are introduced, new energy, smart grid, new ATM procedures, meteorological or weather phenomena, etc.). The System of Interest (SoI) focused in this paper is the vehicle or UAM aircraft and its associated technologies. 


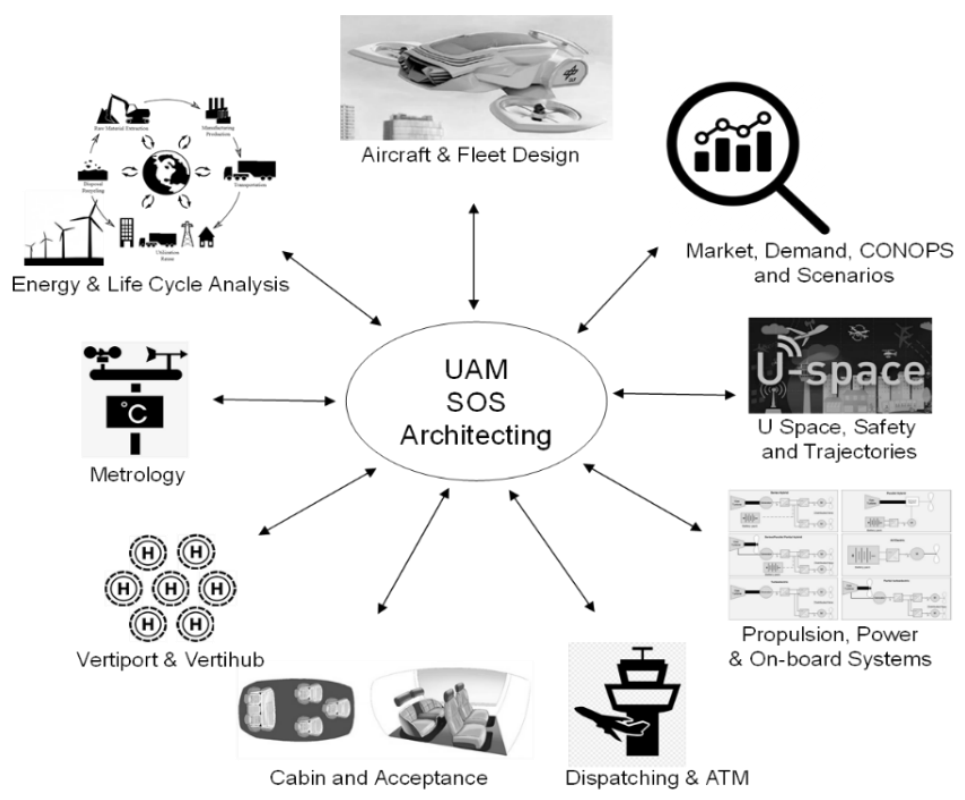

Fig. 2 Urban Air Mobility as a System of Systems

Previous work on UAM aircraft design has provided several comparisons of different architectures relating to overall concepts [14], mission requirements [15], cost as well as acoustics [16], and also sustainability [17]. Researchers have also shown that the concept of operations and thus the mission profile are crucial for well sized eVTOL vehicles [18]. Therefore, simulating the entire UAM network, which is considered a complex SoS, offers the possibility of studying the influences of vehicle design and performance towards the entire SoS capability. While prior research has made use of Agent-Based Simulations (ABS) for UAM to analyze the overall transport network with regard to demand, operational performance, sensitivities [19], and cost optimization [20], only few researchers have addressed UAM aircraft performance and design aspects in the SoS context [21].

Our research proposes a new approach to UAM aircraft design by incorporating a SoS simulation in the aircraft design process in order to address SoI aspects, i.e. UAM aircraft design, in the overall context of transport network and operations management. While a heterogeneous fleet may ultimately be necessary to fulfill the requirements for different UAM missions, this initial paper aims to demonstrate the proof of concept for the newly developed software by simulating scenarios combining UAM aircraft design and operations with regard to a homogeneous fleet. For an assumed demand and vertiport network. An early case study of Hamburg in Northern Germany and California is conducted and analyzed considering two UAM aircraft architectures as well as different technology assumptions.

Several research questions need to be addressed in the UAM SoS for successful design,fleeting and deployment in a given city and for respective operational context. The research questions addressed are the impact of fleet size, payload ( 2 or 4 persons on board), autonomy, powertrain architecture, aircraft configuration, sizing mission, and battery specific energy.

Vehicles of different capacity and architectures need to be designed considering CONOPS for the use cases, the optimal combination of vehicles (fleet mixture) is essential to meet the UAM passenger and network demands. Further, the energy required by the UAM system is an important element to be considered within the SoS. The choice of the propulsion sub-system does not only affect the design of the UAM vehicles, but also determines the re-energizing time of the vehicles. This time strongly impacts the operational throughput, number of passengers transported in the UAM system of systems, the propulsion and recharging subsystem design is therefore an important parameter within the simulation driven design SOS framework.

\section{B. Collaborative Design Process}

The constituent systems in this UAM SoS (see Fig. 3) needs to be orchestrated collaboratively, also even while designing and analyzing these constituent systems, collaborative aspects of design, domain interactions via a common language need to be considered. The SoS framework contains a tool chain across multiple DLR institutes located in different geographical locations and the domain analysis of different constituent systems is performed at different DLR institutes. This desires a collaborative design, uncertainty propagation and robust framework as shown in the 
Fig. 3 below, derived from the lessons learnt from previous European H2020 Project AGILE [22] and collaborative research by the same authors [23], [24], [25].

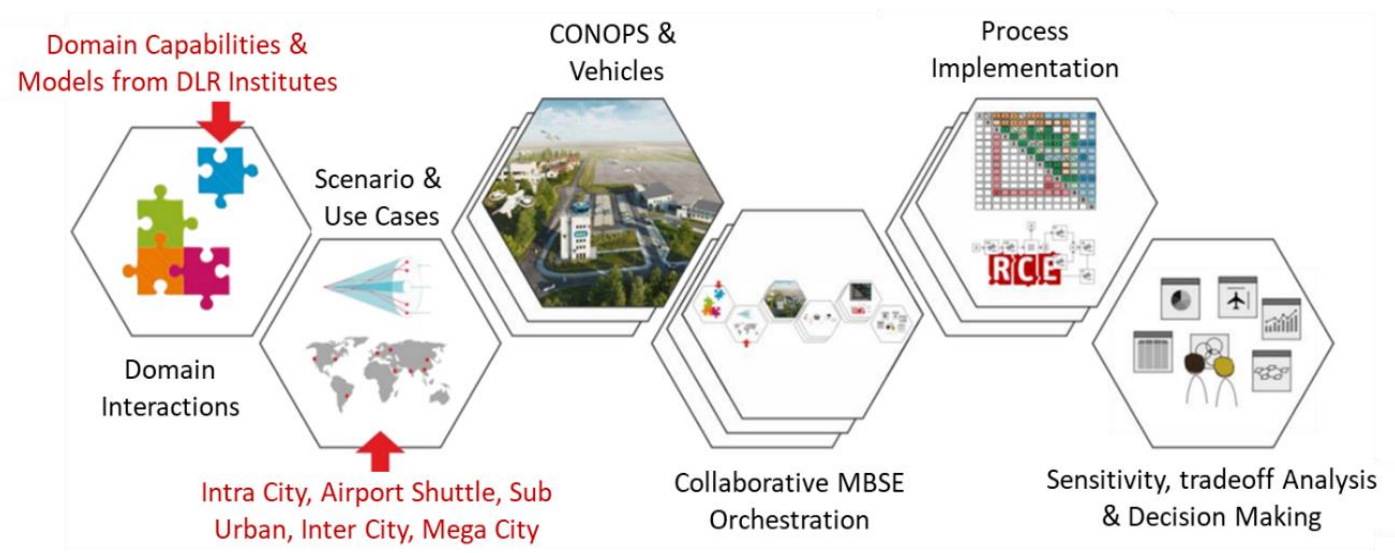

Fig. 3 DLR UAM Collaborative Architecting Process

The System of Systems level evaluation involves heterogeneous vehicle fleet, vertiport, overall fleet level energy lifecycle, average waiting time, mission success based on on-demand availability of UAM vehicle, recharge time etc. The system of Interest level design involves vehicle design and architecture (multirotor, tiltrotor, etc.). The subsystem level design involves power and propulsion subsystems (hybrid-electric, full-electric, etc.). There are interactions between these levels, example: The recharge time depends on the Power and propulsion subsystem, which will impact the vehicle design, this further also effects the mission time or operation speed, number of flights per recharge and waiting time. In the next section we describe a framework to evaluate multi-level research questions.

\section{System of Systems Framework and Methodology}

In order to simulate and evaluate all the systems in the UAM SoS a flexible and extendable SOS framework is developed (see Fig. 4).

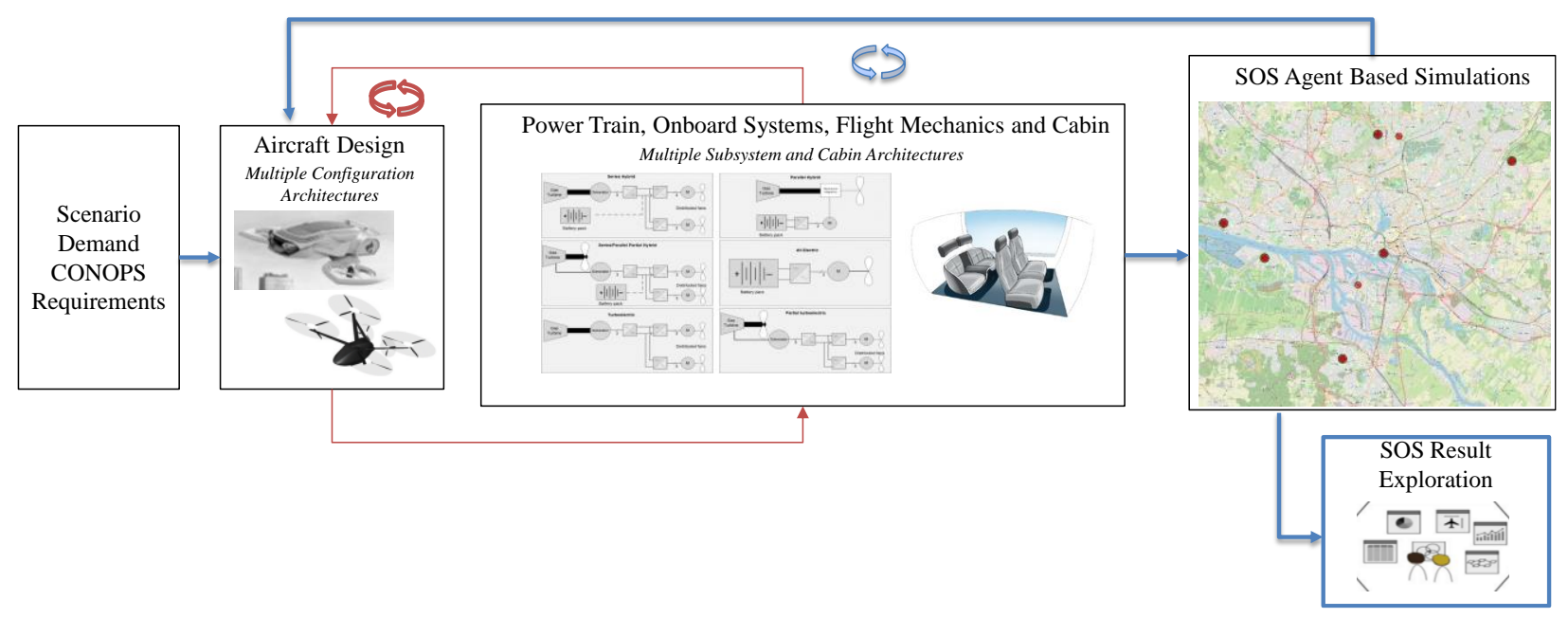

Fig. 4 System of Systems Framework for Urban Air Mobility

This simulation embedded framework accounts for aircraft performance and allows the study of various UAM aircraft architecture specific characteristics, propulsion and onboard system architectures, technology, and operations. Therefore, not only cruise speed and range, but also parameters such as lift-to-drag ratio, disk loading, battery technology, charging power, and the Concept of Operations (CONOPS) can be analyzed. The current implementations are explained in the following sections, where initially the overall simulation framework, then the eVTOL aircraft design tool, and finally the ABS as well as the interaction between both tools is explained. 
The CONOPS, demand, and market studies are part of the publication [26] and the UAM life-cycle assessment is part of the publication in the [27].

\section{A. Aircraft Design}

1. Sizing and Performance

The conceptual aircraft design methodology of this study combines and extends the prior work by Brown and Harris [16] as well as Kadhiresan and Duffy [15].

Here, the rotor model of the open source software for conceptual eVTOL aircraft design presented by Brown and Harris [16] is utilized for the assessment of hover flight performance and is extended by vertical climb as well as forward flight performance. Thereby, momentum theory is applied, where an induced power factor of 1.2 is considered. The assumed rotor parameters are summarized in Table 1. Since a geometric program is used for the rotor sizing, it should be noted that some parameters, i.e. disk loading, blade mean lift coefficient, and blade tip Mach number, are given as optimization constraints.

Table 1 Rotor sizing inputs

\begin{tabular}{lcc}
\hline & \multicolumn{2}{c}{ UAM vehicle configuration } \\
\cline { 2 - 3 } Rotor Parameters & Multirotor & Tiltrotor \\
\hline Disk loading $D L, \mathrm{~N} / \mathrm{m}^{2}$ & $120 \leq D L \leq 180$ & $400 \leq D L \leq 720$ \\
Number of rotors & 18 & 6 \\
Number of rotor blades & 2 & 5 \\
Rotor solidity & 0.1 & 0.1 \\
Blade relative thickness & 20 & 20 \\
Blade zero-lift drag coefficient & 0.01 & 0.01 \\
Blade mean lift coefficient & $\leq 0.6$ & $\leq 1.0$ \\
Blade tip Mach number & $\leq 0.9$ & $\leq 0.9$ \\
\hline
\end{tabular}

Furthermore, Kadhiresan and Duffy [15] have brought together several methods for the conceptual mission analysis of eVTOL aircraft. Herein, the assessment is different for winged compared to wingless eVTOL vehicle configurations. Thus, the aerodynamics of the winged configurations, e.g. tiltrotor, are modeled by a drag polar, where parasite as well as lift induced drag are taken into consideration. In contrast, the forward flight performance of wingless configurations, e.g. multirotor, is assessed by computing the total power, which is a sum of parasite power, induced power, and profile power required at the rotors. In order to estimate the parasite drag coefficient or parasite power coefficient that is required for both, winged and wingless vehicles, the flat plate drag area of the vehicles has to be determined. Respectively, different empirical relations are used, where the flat plate drag area is as a function of maximum take-off mass (MTOM). Consequently, the parasite drag is calculated by the relation of flat plate drag area to the respective reference area of the eVTOL configuration [15]. In this study, the wing sizing is performed with respect to the cruise conditions of the tiltrotor configuration (see Table 2).

Table 2 Wing sizing inputs

\begin{tabular}{lc}
\hline Wing Parameters & Tiltrotor \\
\hline Stall speed $V_{\mathrm{S}}, \mathrm{km} / \mathrm{h}$ & $0.5 V_{\max }$ \\
Wing span, $\mathrm{m}$ & $\leq 15$ \\
Wing aspect ratio & $\leq 15$ \\
\hline
\end{tabular}

The airframe weight estimation in this study again uses the methods summarized by Kadhiresan and Duffy [15]. Depending on the configuration, different structural weight groups are estimated, which means that a wingless configuration only consists of fuselage and landing gear, whereas wing and empennage are added in case of the winged configuration. With regard to fuselage sizing it should be noted that two different cabin geometries are assumed for a 2- and 4-seater, which is related to the later described Top Level Aircraft Requirements (TLAR) in Table 4. Accordingly, the wetted area of the fuselage can be determined for the weight estimation. 
Finally, by the combination and extension of the previously explained methodologies, the following UAM aircraft design loop is set up (see Fig. 5). The sizing procedure of powertrain and onboard systems, i.e. the subsystem level of this study, is thoroughly explained in the following Section II.B. Moreover, the connection of both tools is discussed in Section II.D.

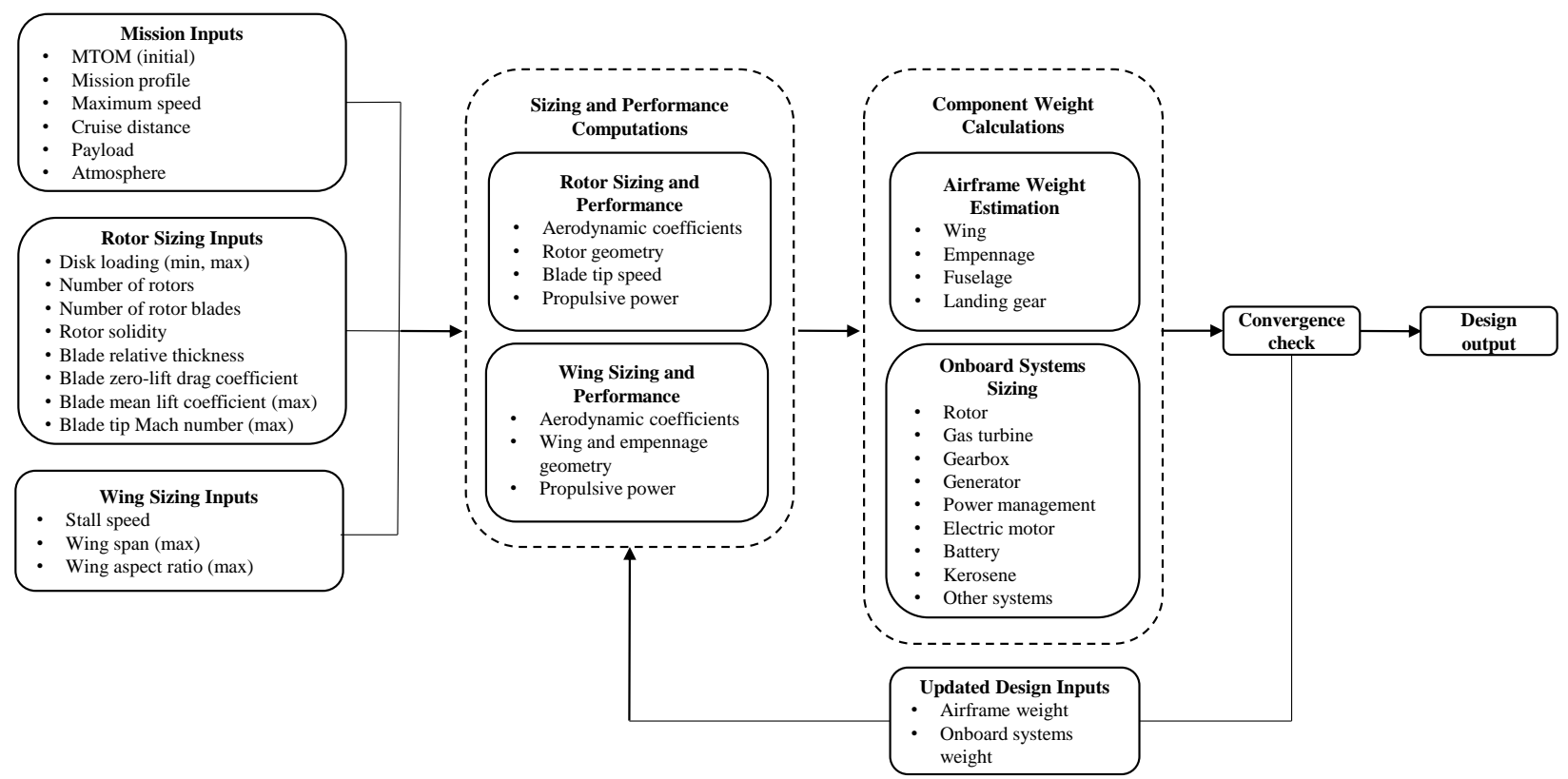

Fig. 5 Sizing Loop for initial UAM aircraft design used in this study

\section{Top Level Aircraft Requirements and Mission Definition}

In this study, two different sizing missions are considered, whereby the multirotor is sized for a shorter cruise distance compared to the tiltrotor. However, the same mission profile is utilized (Fig. 6). A detailed summary of the underlying mission segment definitions is presented in Table 3. The mission profile has been set up with reference to other researchers within the field of UAM aircraft design [28]. Additionally, a headwind of $50 \mathrm{~km} / \mathrm{h}$ is considered in the forward flight segments.

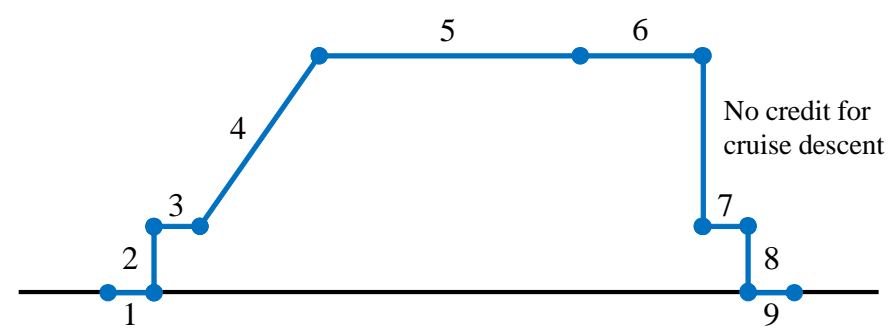

Fig. 6 Sizing mission profile 
Table 3 Sizing mission segment definitions

\begin{tabular}{lcccccc}
\hline Segment & $\begin{array}{c}\text { Forward } \\
\text { speed }\end{array}$ & $\begin{array}{c}\text { Vertical } \\
\text { speed, fpm }\end{array}$ & $\begin{array}{c}\text { Altitude } \\
\text { AMSL, ft }\end{array}$ & Distance & Time, min & Power \\
\hline 1 Start up & - & - & 0 & - & 0.3 & $0.1 P_{\mathrm{cr}}$ \\
2 Vertical climb & - & 100 & 0 to 50 & - & 0.5 & $P_{\mathrm{vc}}$ \\
3 Transition & 0 to $1.2 V_{\mathrm{S}}$ & - & 50 & - & $t_{\mathrm{tr}}$ & $P_{\mathrm{h}}$ \\
4 Cruise climb & $V_{\mathrm{mp}}$ & 750 & 50 to 1500 & $D_{\mathrm{cc}}$ & 2 & $P_{\mathrm{mp}}$ \\
5 Cruise & $V_{\mathrm{md}}$ & - & 1500 & $D_{\mathrm{req}}-D_{\mathrm{cc}}$ & $t_{\mathrm{cr}}$ & $P_{\mathrm{md}}$ \\
6 Loiter & $V_{\mathrm{mp}}$ & - & 1500 & - & 20 & $P_{\mathrm{mp}}$ \\
7 Transition & $1.2 V_{\mathrm{s}}$ to 0 & - & 50 & - & $t_{\mathrm{tr}}$ & $P_{\mathrm{h}}$ \\
8 Vertical descent & - & -100 & 50 to 0 & - & 0.5 & $P_{\mathrm{vc}}$ \\
9 Shut down & - & - & 0 & - & 0.3 & $0.1 P_{\mathrm{cruise}}$ \\
\hline
\end{tabular}

In this study, two different use cases, namely urban and suburban, are considered. Each of the use cases is fitted to the UAM vehicle configuration characteristics. Subsequently, the TLAR are summarized in Table 4. Here, the term payload actually refers to the number of Persons On Board (POB), which means that 2 POB require a 2-seater, and $4 \mathrm{POB}$ require a 4-seater, respectively. In a near-term scenario, where autonomous operations may not be fully deployed, a pilot will occupy one of the passenger seats. Still, the payload in the sizing mission remains the same, since the mass per person is assumed to be $90 \mathrm{~kg}$ irrespective of pilot or passenger.

Table 4 Top level aircraft requirements

\begin{tabular}{lcc}
\hline & \multicolumn{2}{c}{ UAM vehicle configuration } \\
\cline { 2 - 3 } Requirement & Multirotor & Tiltrotor \\
\hline Maximum speed $V_{\max }, \mathrm{km} / \mathrm{h}$ & 100 & 270 \\
Headwind, $\mathrm{km} / \mathrm{h}$ & 50 & 50 \\
Distance $D, \mathrm{~km}$ & 30 & 70 \\
Reserve time, min & 20 & 20 \\
Persons on board & 2 & 4 \\
Payload mass, $\mathrm{kg}$ & 180 & 360 \\
\hline
\end{tabular}

Finally, the aircraft performance is calculated for all possible mission segments and load factors. Here, the power required is a constant value for each segment. These performance outputs are then used within the SoS Agent-Based Simulation (see Section II.C). Also, it should be noted that the UAM vehicles are flown at the design best range speed during cruise climb and cruising flight in the simulation, which is accounted for by the described performance computations.

The results of the aircraft design and performance, which are based on the onboard systems results, are presented in Section IV.B. 


\section{B. Propulsion and Onboard System Architectures}

In addition to the mission- and performance-based calculations, the design of the onboard systems is another important building block in the eVTOL design. The onboard system consists of various subsystems such as flight control, avionics, etc. with a large number of components. The design goal in this study is to determine and investigate the system weight based on the propulsive power requirements. The system weight is given back to the aircraft design level in order to iteratively determine the total aircraft weight.

\section{Propulsion System Architecture Model}

In the following, an analytical, steady-state model for the design of (hybrid-)electric powertrain architectures is presented, which is based on the work of de Vries [29]. This model is used to calculate the power of each individual powertrain element for a given propulsion power requirement. The drive power of the eVTOL is determined via the mission profile and made available to the model (see section A).

a) Serial Hybrid-Electric Powertrain Architecture

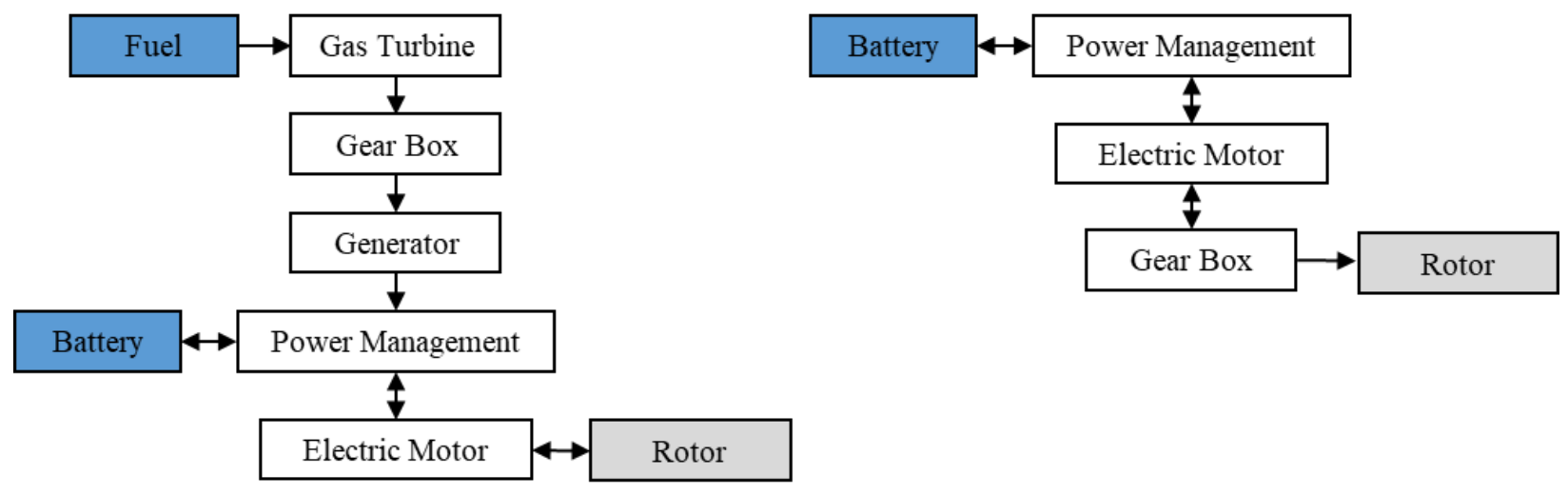

b) Full-Electric Powertrain Architecture
Fig. 7 Powertrain architectures investigated in this study

In this study, both a fully electric and a serial, hybrid-electric powertrain architecture will be investigated, which can be seen in Fig. 7. In addition to the known propulsive performance requirements, the model also has to deal with various hybrid- and fully electric powertrain architectures. De Vries proposed various architectures for his model [29]. His investigation showed that the so-called serial / parallel partial hybrid (SPPH) architecture can be used as a general model to configure and calculate other hybrid- and fully electric architectures via two power control parameters (supplied power ratio $\Phi$, shaft power ratio $\varphi$ ).

Especially the supplied power ratio $\Phi$ is relevant for the serial architecture. It represents the amount of energy taken from the electrical energy source (batteries) in relation to the total amount of energy that is taken from all energy sources (in this case battery plus fuel) for a certain point along the mission. In this work, the proportion of electrical power drawn for the different eVTOL concepts and mission sections is constant at $20 \%(\Phi=0.2)$. With the aid of these power control parameters, a subsequent analysis and optimization of the power settings during the mission can also be made possible [29] However, that is not part of this study.

The powertrain model consists of a total of ten unknowns that correspond to the power paths of the general model. Therefore, ten equations are needed to solve the system (see [29]). In addition to the equations for the power control parameters and another equation for dividing the total flight performance into two different, propulsive paths, there is an equation for the power balance of each of the seven possible drive train components.

In order to establish the balance between the incoming and outgoing power quantities of a component, the corresponding component efficiency $\eta$ is specified. Table 5 gives an overview of the efficiencies used in this study.

$$
\sum P_{\text {out }}=\eta \cdot \sum P_{\text {in }}
$$

The created linear system of equations is finally solved by entering the flight performance requirement for each flight phase. As a result, the power parameters of the individual components are calculated for each phase. The component weights are finally calculated using the component specific power (SP) values (or power-to-weight ratio). 
The values used for each individual component are shown in Table 5. Remark: the rotor efficiency is only considered for propeller driven aircraft.

$$
m_{\text {comp }}=\frac{P_{\text {comp }, \text { max }}}{S P_{\text {comp }}}
$$

In contrast, the calculation of the fuel weight is not based on power but on energy requirements. In addition to the required power, the duration of the respective power output is determined directly via the mission profile. The weight calculation is ultimately based on the specific energy (SE) of 11,800 Wh/kg (for kerosene).

$$
m_{\text {fuel }}=\frac{E_{\text {fuel }}}{S E_{\text {fuel }}}
$$

The battery weight calculation is based on both power and energy requirements and is therefore different from the other components of a (hybrid-)electric powertrain. The battery mass is ultimately determined from the maximum value of both requirements.

$$
m_{\mathrm{bat}}=\max \left(\frac{P_{\mathrm{bat}, \mathrm{max}}}{S P_{\mathrm{bat}}}, \frac{E_{\mathrm{bat}}}{S E_{\mathrm{bat}}}\right)
$$

Other important parameters such as state of charge (SoC) or the system voltage have been neglected for simplification in the battery model (battery efficiency of 0.96), except the minimum and maximum depth of discharge (only $80 \%$ of total capacity is used). In addition to the C-rate, which is constrained by a maximum of $2.5 \mathrm{C}$, the values for the battery specific energy are varied in this study in order to take various technological advances into account (see Table 5).

Table 5 Component specific power and efficiency

\begin{tabular}{lcc}
\hline Powertrain component & Specific power, kW/kg & Efficiency \\
\hline Gas turbine & 8.2 & 0.50 \\
Gear box & 5 & 0.98 \\
Rotor & 10 & 0.80 \\
Generator & 4 & 0.95 \\
Power Management & 5 & 0.98 \\
Electric Motor & 4 & 0.95 \\
\hline
\end{tabular}

\section{Model for Other Onboard Systems}

While it is possible to consider different powertrain architectures for the eVTOL design, the other systems such as flight control, avionics, instrumentation, environmental control system, and furnishings are summarized in a general model, which was taken from [30].

$$
m_{\text {sys, other }}=0.0239 \cdot M T O M+195.71
$$

This model requires the aircraft MTOM as an input variable in order to determine the system weight using a linear approach. It should be noted that the model works with the unit [lb] and therefore the input and output values for this work are converted into the unit [kg]. In the future, the modeling of these onboard systems is to be improved, for example by considering the systems as a mission-dependent, electrical load in the design of the powertrain.

Eventually, results and discussions of the propulsion and onboard subsystems are presented in the later following Section IV.A. 


\section{Agent-Based Simulation}

This section explains the DLR in-house ABS for analyzing a complex SoS. The ABS framework is used to power the UAM example. For the development of the ABS framework, please refer to [32]. The ABS for UAM is composed of two main models, the demand model and the agent model with additional methods and classes defined for implementation of the desired features as shown in Fig. 8Fig. 8 UAM Agent Based Simulation Approach. The simulation framework was developed with an emphasis on modularity. As such, the capabilities to simulate any desired city, region or country with ease is incorporated.

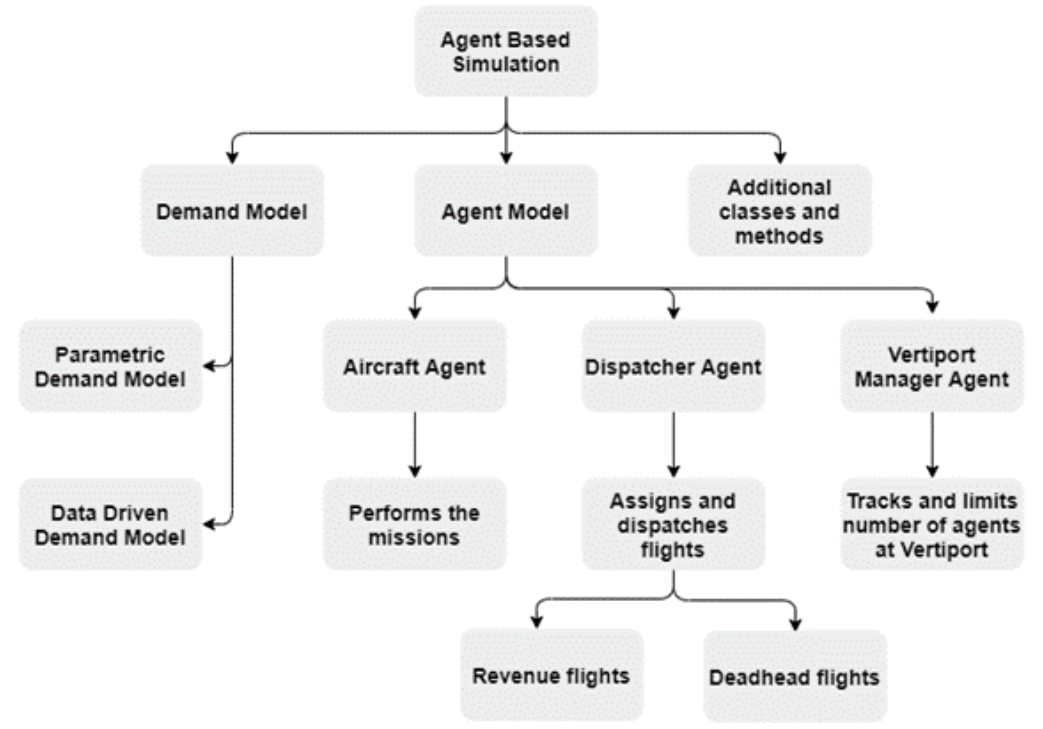

Fig. 8 UAM Agent Based Simulation Approach

\section{Demand Model}

In the ensuing discussion, the term `demand is used synonymously with passenger or mission. It is also used to refer to a single passenger demand or the overall demand of the network. A deadhead flight or mission is a nonpassenger carrying flight flown to reposition the aircraft. The term `deadhead demand therefore refers to a deadhead mission. In addition, a revenue flight or mission is a passenger carrying flight.

In the development of the demand model, special care was given to ensure it is modular to allow for quick modification of the demand model based on available data. The demand model consists of inflow and outflow curves defined at each vertiport. The inflow and outflow curves consist of multiple normal curves which make up the desired distribution. The normal curves are described by the peak demand magnitude and the hour at which the peak occurs in addition to its standard deviation. In the current study, the standard deviation is kept constant at 2 hours. The outflow and inflow curve descriptions are then discretized into hour-long segments with their respective demand magnitudes evaluated at the middle of the hour-long segment.

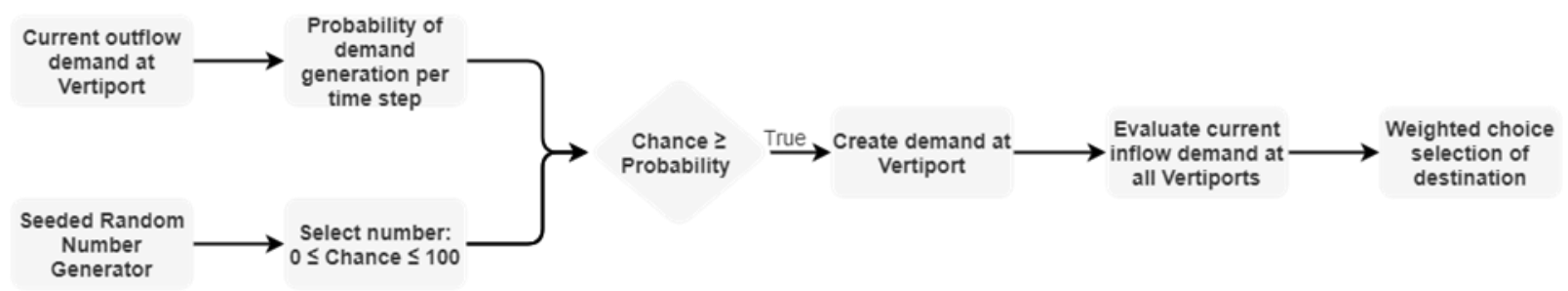

Fig. 9 Demand generation logic

Fig. 9 describes the logic behind the demand generation, which is applied at each iteration of the simulation for each vertiport. Initially, the value of the discretized outflow demand at the simulation time is retrieved and converted to a probability of demand generation per time step. This probability is then compared to a chance value selected by a seeded random number generator between 0 and 100. If the chance is greater than the probability, a demand is 
generated at the vertiport. The destination of the generated demand is chosen by a weighted choice selection of the vertiports considering the inflow demand magnitudes at the simulation time for each vertiport. From this point, the agent model is tasked with the assignment and dispatching of agents to fulfill the mission.

This work provides a proof of concept of the SoS driven aircraft design framework by investigating the impact subsystem level variables propagated through the SoI level to the SoS level for an assumed demand model. As the demand for UAM operations is not well known in literature, the demand inputs used in this study are informedassumptions. A seeded RNG is used to ensure the stochasticity is deterministic and reproducible. In the future work, data driven demand will be incorporated in the investigations, as soon as it becomes available.

\section{Agent Model}

The agent model consists of the aircraft agents, the dispatcher agent, and the vertiport manager agents. The fleet definition consists of defining the number and characteristics of the aircraft agents. The cruise speed, passenger capacity, technology assumptions and performance are among the characteristics that can be defined for the aircraft agents. The fleet level definition consists of the size, and initial distribution of the aircraft agents. In this study, only homogenous fleets are considered and the deployment of heterogenous fleets is left for future studies.

The dispatcher agent assigns and dispatches the revenue as well as deadhead flights to the aircraft agents. The mission assignment is carried out using a bidding model in which each aircraft agent submits its bid for the mission in consideration. The dispatcher assigns each mission to the aircraft agent with the highest bid. The bidding model was developed with the aim of maximizing the percentage of successful missions and reducing the number of required deadhead flights by the assignment of each mission to the ideal aircraft agent based on the set criteria. After receiving a new demand, the dispatcher calls on all aircraft agents to place their bids, which consist of three parameters: the number of passengers assigned to the same mission, the estimated time of completion of the mission, and the available energy of the agent. In the computation of the estimated time of competition of the new demand, the agent considers the time needed to complete the active mission, if any, and any required charging time. The parameters considered in the bidding model are normalized by the time taken to fly the furthest distance between any two vertiports, the maximum passenger capacity, and the total battery capacity respectively. Each term in the bid equation 6 is multiplied by a weighting factor, the weights of each term were chosen with an order of magnitude difference to represent the priorities set for the dispatching. Namely, the first priority is to maximize the load factor of the flights, secondly to reduce the wait time of the passengers and lastly to use aircraft with higher instantly available energy. Together with the normalization, the order of magnitude difference in the weighting ensures a hierarchical decision-making process in accordance with the dispatch priorities.

$$
\text { bid }=w_{1} \cdot \frac{n_{\text {assigned passengers }}}{n_{\text {passenger capacity }}}+w_{2} \cdot \frac{t_{\text {mission completion }}}{t_{\text {longest route }}}+w_{3} \cdot \frac{E_{\text {useable energy }}}{E_{\text {battery capacity }}},
$$

where $w_{1}=10, w_{2}=1$, and $w_{3}=0.1$.

Each aircraft agent can be assigned up to 2 missions at once, while the missions are carried out sequentially. Once an aircraft agent wins the bid and is assigned the mission, the mission is then allocated to one of these slots if possible. The allocation logic is described in Fig. 10. In this study, the deadhead demands are only generated once necessary: in the cases where the agent to which the mission was assigned to is not in the same vertiport as the demand, in such a case a deadhead demand is dispatched to transport the agent to the required vertiport. The network imbalance requires non-revenue "deadhead" flights to re-distribute aircraft from the areas of low demand to areas of high demand [33]. 


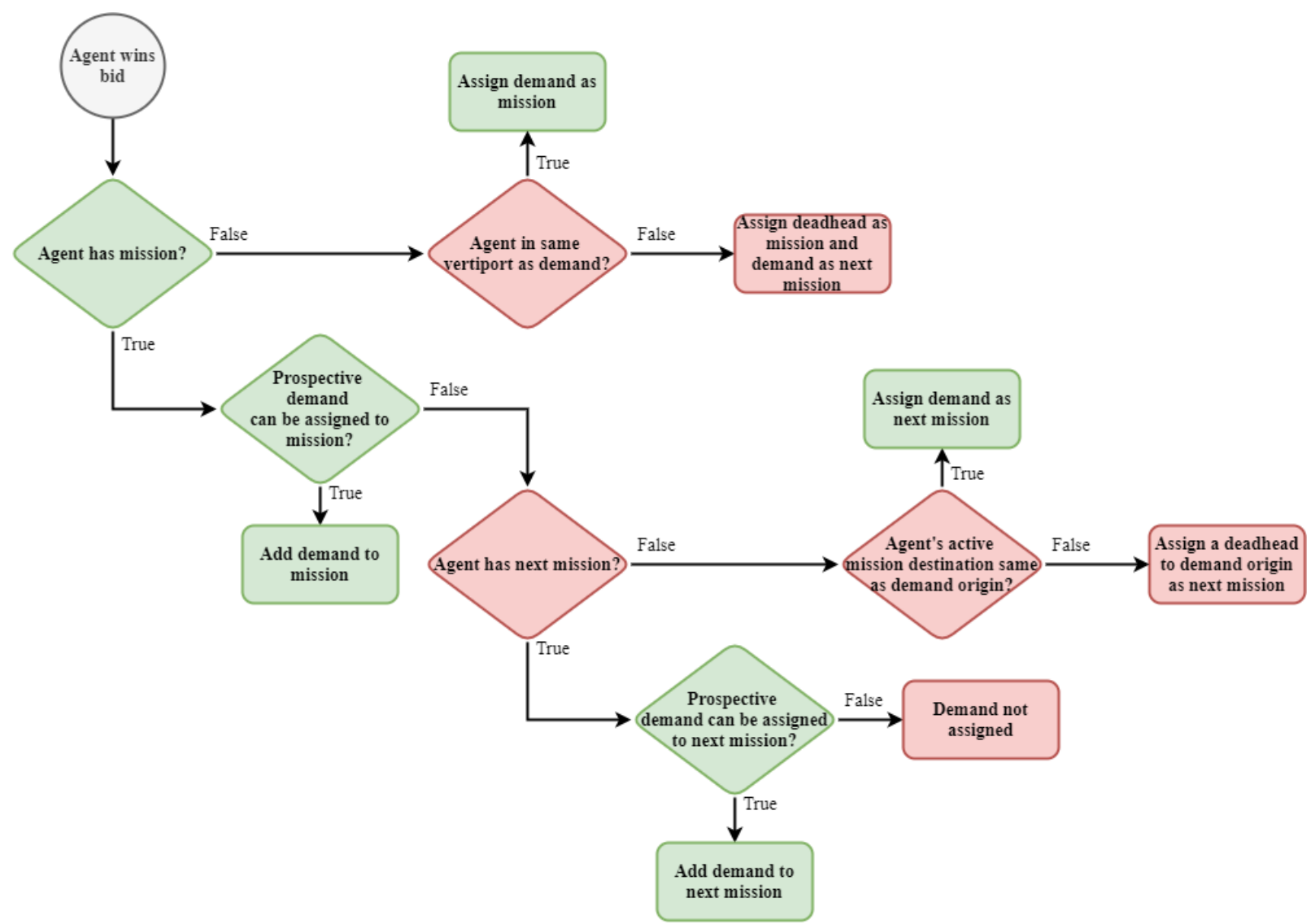

Fig. 10 Logic for demand allocation to aircraft agent

Once the mission is assigned, the aircraft awaits dispatch by the dispatcher. If the assigned mission of the aircraft agent is a revenue mission, then the aircraft agent is dispatched when the dispatch criteria is satisfied. The dispatch criteria to satisfy is either the wait time of any demand assigned to the aircraft reaching the target wait time, or the aircraft agent reaching a load factor of 1 . The aircraft agent is made to wait to allow for any possible additional demand to be assigned to it, thereby increasing the load factor. If the assigned mission is a deadhead mission, then the aircraft agent is dispatched immediately. In the special case that the agent is assigned a deadhead mission as its next mission and a revenue demand is generated for the same route as the deadhead prior to the dispatching of the deadhead mission, the revenue demand can be assigned to the scheduled deadhead mission, thereby allowing the operator to get revenue from the scheduled deadhead mission when possible.

The vertiport manager agent has two objectives: managing the number of agents at the vertiport and giving permission for takeoff and landing. In this study however, the limit on the vertiport capacity is not applied.

\section{Energy Model}

The energy model in the simulation is tasked with tracking and updating the energy consumption and the reenergizing of the vehicle. From the sizing tool, the power consumptions for the revenue, deadhead and sizing missions are computed. The power consumptions of the revenue mission are computed for each possible load factor. Moreover, for both the deadhead and revenue missions, the powers associated with each mission segment is provided to the simulation from the sizing tool. In each iteration of the simulation, the available energy of the aircraft is updated based on its state of flight and its load factor. The simplifying assumption of constant power usage is assumed within each state of flight and load factor. In addition, the aircraft agents are assumed to charge their batteries at a constant rate defined by the charging power input when at a vertiport. Here, a charging efficiency of $90 \%$ is considered. All aircraft agents are assumed to start the simulation with a fully charged battery, that is the total battery capacity reduced by the usable energy fraction and energy required for the reserve mission. 


\section{Connection of Aircraft Design, Propulsion and Onboard Systems, and Agent-Based Simulation}

The remote component environment, RCE, was utilized for the aircraft design presented in this study. The UAM aircraft sizing tool and the onboard systems sizing tool were connected and the design loop executed as presented in Fig. 5. The final design outputs were then input into the ABS through a Design of Experiments (DoE) alongside the use-case definition. The DoE runner module then executes the DoE with multi-processing and outputs the data for each design point.

\section{E. Limitations of the UAM System of Systems framework}

This framework is the first attempt to evaluate the UAM use case from Subsystem over System of Interest to the System of Systems level. Currently, there are certain limitations:

1) The framework assumes homogeneous fleets, where each aircraft agent is of the same type and characteristics, e.g. cruise speed, payload, etc. The capability of simulating heterogeneous fleets exists, but the dispatch and deadhead modelling needs to be optimized considering different passenger capacities, weightage factors and performance characteristics.

2) The framework assumes pseudo passenger demand and unlimited vertiport landing pads and parking/gate capacity. The UAM passenger demand can be included when there is a broader study of market and vertiports. The limited landing pads and parking capacity will require further optimization with regard to dispatching.

3) The trajectories assumed between vertiports is a simple direct flight consisting of the mission segments, as shown in the mission profile (see Fig. 6). Ideally, the trajectories should be modelled considering important factors such as noise disturbances and airspace clearances among others, i.e. modelled closer to what is expected in reality. More detailed work will be carried out as explained in the dedicated paragraph on future work (see Chapter V).

\section{Case Study}

To evaluate the SoS framework two example use cases of Hamburg, Germany (City of Hamburg) for multirotor with average mission range of $15 \mathrm{~km}$ and Northern California, USA (San Francisco Bay Area) for tiltrotor with average mission range of $35 \mathrm{~km}$ is considered. To evaluate the UAM SoS framework for any given city, multilevel sensitivity is needed. For which, the market or passenger demand is needed. Since there is no extensive study available and considering the inherent uncertainty of the market adoption of UAM, the authors decide to assume a demand which is parametric and changeable for any new inputs of demand data. Secondly, the vertiport locations should be derived based on the city topography, demand, and intermodal transport. The vertiport locations are assumed for this current study and can be easily varied in the framework using latitude and longitude coordinates when the study is updated with market studies. Fig. 11 shows the demand distribution at six vertiports, both assumed, for the city of Hamburg in Northern Germany. The average distance between the vertiports is approximately $15 \mathrm{~km}$. It is worth note that all missions are flown at the best range speed of the aircraft.

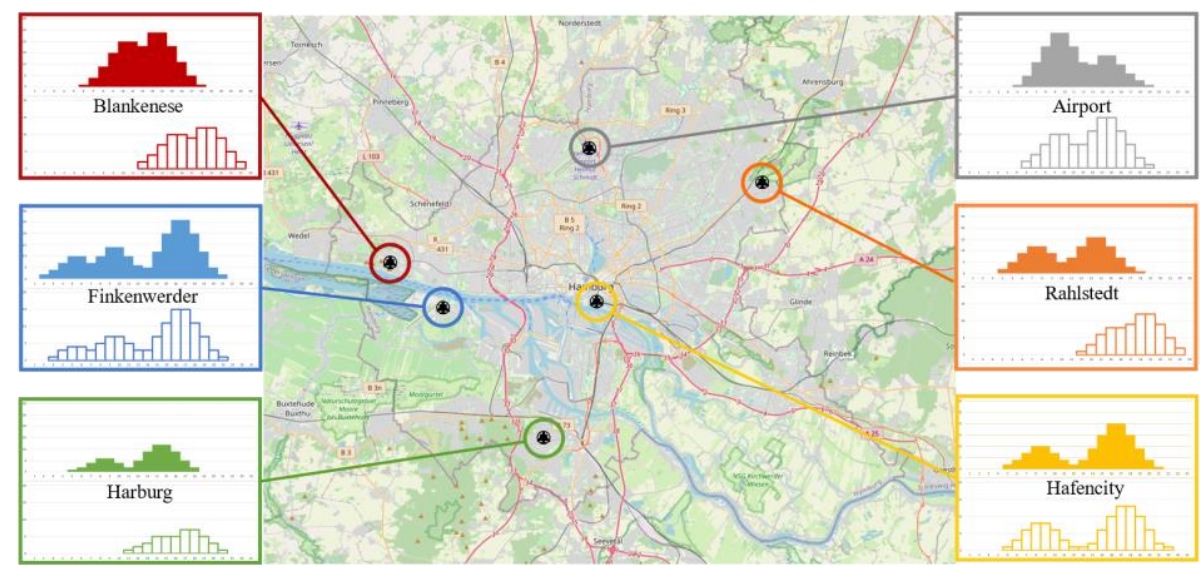

Fig. 11 UAM use case for Hamburg, Germany, showing vertiport locations and corresponding demand (outflow in solid color, inflow with no fill) 


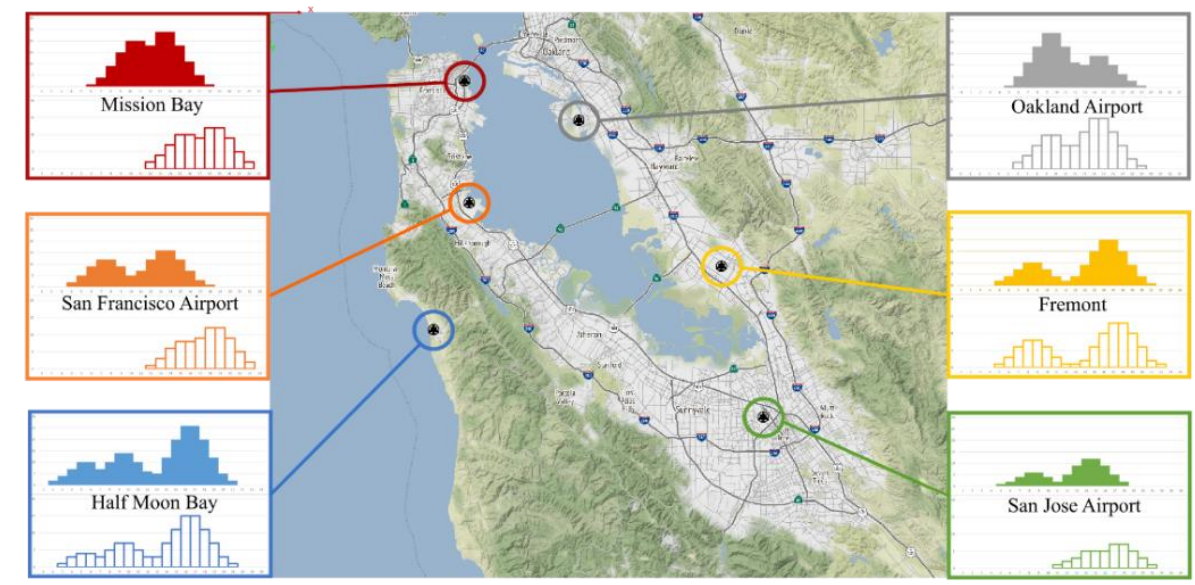

Fig. 12 UAM use case for San Francisco, USA, showing vertiport locations and corresponding demand (outflow in solid color, inflow with no fill)

Table 6 Summary of the case study parameters

\begin{tabular}{lcc}
\hline Case study parameter & Count & Design points \\
\hline Aircraft & 2 & Multirotor, tiltrotor \\
Sizing missions & 2 & Urban (2 POB, 30 km), suburban (4 POB, 70 km) \\
Powertrain architectures & 2 & Hybrid-electric, full-electric \\
Sizing missions & 2 & Urban (2 POB, 30 km), suburban (4 POB, 70 km) \\
Reserve requirement & 1 & $20-$-minute loiter \\
Battery pack specific energy, $\mathrm{Wh} / \mathrm{kg}$ & 3 & $250,400,550$ \\
Battery max. discharge C-rate, $1 / \mathrm{h}$ & 1 & 2.5 \\
Battery max. charge C-rate, $1 / \mathrm{h}$ & 1 & 2.4 \\
Passenger demand & 1 & Distributions scaled to a maximum of 48 revenue pax per hour \\
Homogeneous fleet size & 1 & $12,18,24,30,36,42,48,54,60,66,72,78,84,90,96$ \\
Vertiport capacity & 1 & 100 (see Section II.E) \\
Target wait time, min & 1 & 15 \\
\hline
\end{tabular}

The success criteria considered for the mission is the wait time of the passengers compared to the target wait time. In this study the target wait time is defined as 15 minutes, which means that each passenger waiting more than 15 minutes is considered a failed mission. This value is set allowing enough time for the passenger to reach the vertiport from their location through other modes of transport. The percentage of successful missions is used as the primary factor for gauging the success of the SoS, and is hereby referred to as the success percentage. Approximately 2250 runs spanning 450 design points and 5 seeds were analyzed in this DoE and particular trends are shown in the following. It is important to note that in the presented results, the data is averaged over the 5 seeds to ensure the stochastic effects are minimized.

The parameters shown in the results of this study are explained in the following:

- Wait Time - Elapsed time from demand creation in the simulation until take-off

- Revenue Passenger Transported - Total number of passengers transported in a given 24-hour operations

- Average Wait Time - Average wait time of all revenue passengers

- Success Percentage - Percentage of revenue passengers waiting less than target time of 15 minutes

- Deadhead ratio - Ratio of deadhead flights (non-passenger carrying flights) to passenger (revenue) flights

- Load Factor - Average load factor of all revenue and deadhead flights (computed excluding the pilot)

- Energy used by network - Total energy used by the UAM SoS or network 


\section{Results and Discussion}

As shown in Fig. 13, below, the result sections, observations made from onboard and power train (subsystem) level, aircraft (system of interest) level, and fleet (system of systems) levels are expressed. Further, the results at Systems of Systems levels are addressed in the form of research questions and discussions. While some are from the point of view of the SoS, others are from system of interest or subsystem perspective. Each question emphasizes the need for analyzing UAM from a SoS perspective.

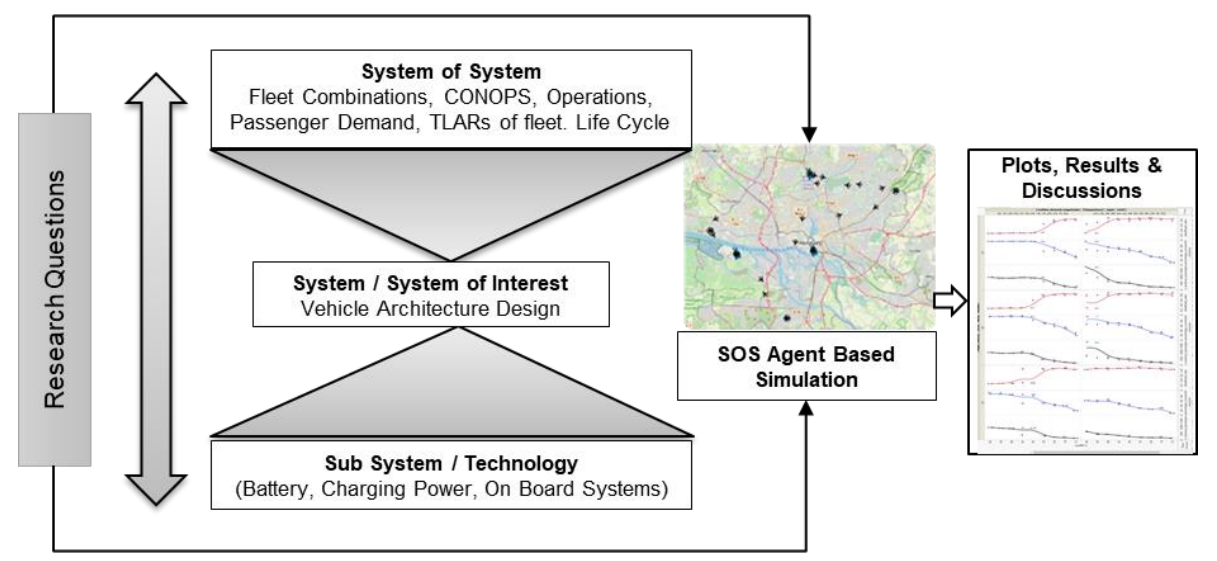

Fig. 13 Multi-level research questions and discussions

Initially, Section IV.A contains onboard systems (subsystem) results. Next, Section IV.B contains aircraft design (system of interest) results. Finally, the research questions highlight the interactive coupling between subsystem, system of interest, and system of systems levels:

1) What is the impact of autonomy on the required fleet size for multirotor in urban Hamburg use case?

2) What is the impact of autonomy on the required fleet size for tiltrotor in suburban California use case?

3) What is the impact of hybrid- and full-electric powertrain architecture on the required fleet size for multirotor in urban Hamburg use-case?

4) What is the effect of battery pack specific energy on the energy consumed by the fleet under the consideration of hybridization and autonomy?

\section{A. Onboard Systems (Subsystem) Results}

In the following, the results of the onboard systems weight are analyzed in more detail. Based on the defined study, two different aircraft configurations (tilt rotor, multirotor) with two different electric powertrain architectures (fullelectric, serial hybrid-electric) are examined. Three battery technology levels $(250,400$, and $550 \mathrm{Wh} / \mathrm{kg})$ are considered, so that there are six variants per aircraft configuration. These six variants for both aircraft configurations are shown in Fig. 14. It can be seen that the weight decreases with improved battery technology. This characteristic is more pronounced in the fully electric powertrain system than in the hybrid-electric variant. This also means that the potential for improvement in terms of system weight is greater in a fully electric system. However, one must consider that only $20 \%$ of the energy, which is needed by the hybrid-electric powertrain in this study, is taken from the battery (see Section II.B, supplied power ratio). A different supplied power ratio would therefore result in a different curve. Investigating this, however, is not part of this work. 


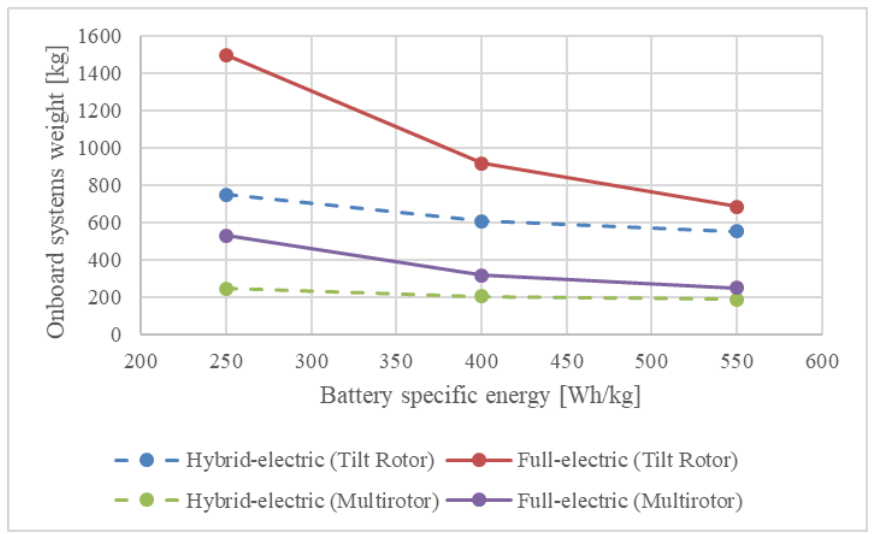

Fig. 14 Onboard systems weight for both eVTOL configurations and powertrain architectures for different battery technology levels

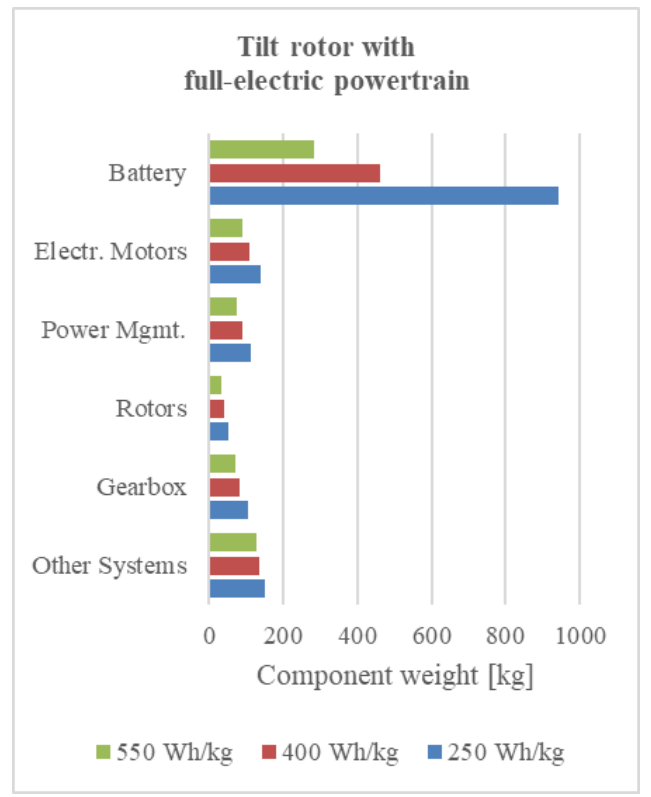

Fig. 15 System component weights for tilt rotor with full-electric powertrain and different battery technology levels

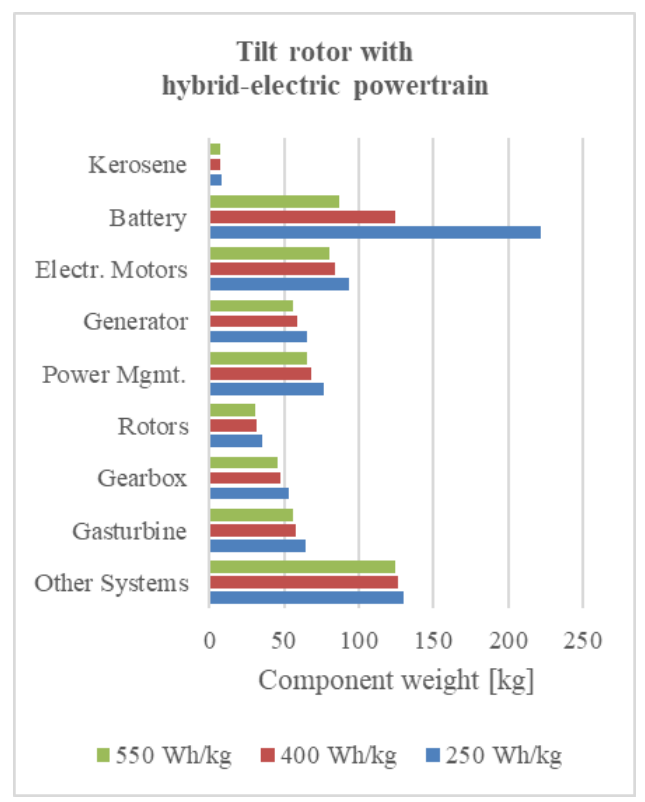

Fig. 16 System component weights for tilt rotor with hybrid-electric powertrain and different battery technology levels

The absolute values of the system weights in the curves of Fig. 14 correspond to the values that were used in the SoS simulation. In order to get a more precise understanding of how the total weight of the individual variants is made up, the component weights for the variants of the tilt rotor are examined more closely. For a better overview, the tilt rotor with a full-electric system is shown in Fig. 15 and the tilt rotor with hybrid-electric powertrain in Fig. 16. In almost all variants it can be seen that the proportion of the battery weight is the highest. Since the battery technology parameter specific energy was varied, a high weight saving for the battery could be expected.

In comparison, the weight savings in the other system components, for which no technological changes have been assumed, are relatively small. This change in weight can be explained by the fact that the eVTOL has an overall low take-off or empty weight and thus the required power requirement for the mission decreases. This lower flight performance is propagated to all powertrain components via the linear system model, which is ultimately the input variable for component sizing (see Section II.B). 


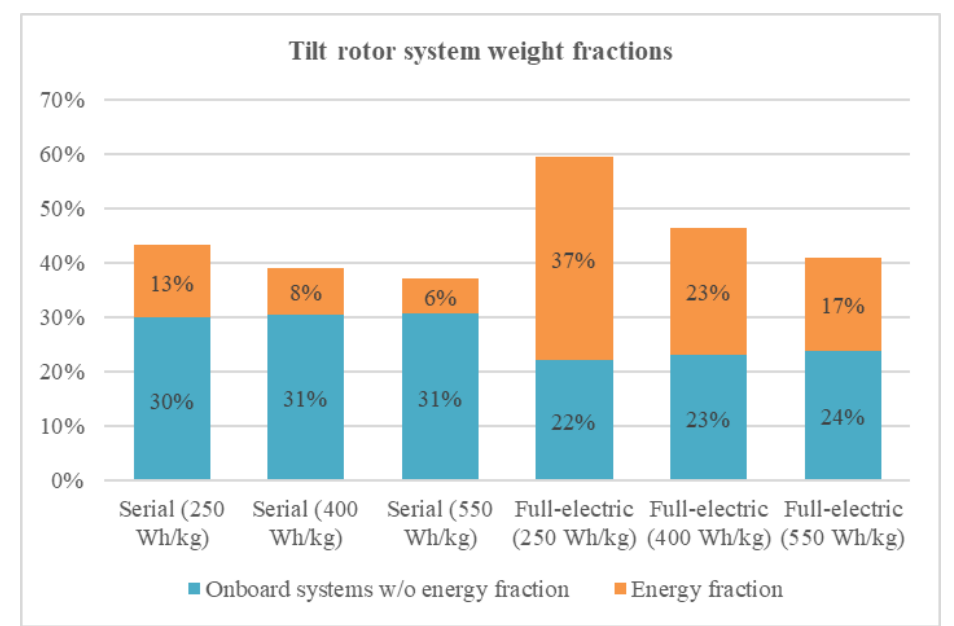

Fig. 17 Relative onboard system and energy weight fraction evolution for the tiltrotor configuration

To verify these findings, Fig. 17 shows the development of the relative weights for the onboard systems, consisting of the fractions for the energy sources (battery, kerosene) and the actual systems (without energy sources). These fractions were related to the respective total aircraft weight. The analysis shows, on the one hand, the comparable, decreasing tendencies of both systems as in Fig. 17. On the other hand, however, it becomes clear that the significant weight savings are caused by the improved battery technology in the area of energy sources. In the case of a fullelectric powertrain system, for example, the fraction of the energy source (here: battery) with improved technology drops from $37 \%$ to $17 \%$. In comparison, the ratio of the onboard systems (without an energy source) to the aircraft weight remains almost constant, which in turn can be explained with the linear system model described above. These results can be seen for both the tilt rotor and the multi-rotor, which has different operational and performance requirements (multirotor results in the appendix)

With the analysis, the following findings can be recorded for the onboard systems and their influence on the eVTOL design. The choice of the architecture of the powertrain system has a significant influence on the total weight of the onboard systems. Two significantly different architectures, a full- and a hybrid-electric, were examined. These architectures showed great differences in terms of the absolute system weight. The absolute system weight also depends heavily on the technology used. On the one hand, the better the technology used, the smaller can be the differences between different powertrain architectures. On the other hand, the absolute system weight can also be greatly reduced. Furthermore, the system weight has a relatively large influence on the aircraft weight. In the context of this study carried out, proportional and technology-dependent system weights of around $40-60 \%$ related to the aircraft weight could be identified (see Fig. 17). 


\section{B. Aircraft Design (System of Interest) Results}

Regarding the system of interest level, several results that highlight the sensitivities due to advancing battery technology will be presented and discussed. Here, findings on the resulting MTOM, mass fractions, and airframe mass breakdowns of both eVTOL vehicles and powertrain architectures are shown.

Initially, Table 7 summarizes several vehicle parameters of both eVTOL configurations and powertrains at a battery pack specific energy of $400 \mathrm{Wh} / \mathrm{kg}$ in order to provide a brief overview of sizing requirements and results. It should be noted that the battery sizing of the full-electric tiltrotor is driven by the maximum discharge C-rate of $2.5 \mathrm{C}$. Furthermore, it can be found that, considering a charger efficiency of 0.9 , a relatively high charger power of $492 \mathrm{~kW}$ is required for fast charging at $2.4 \mathrm{C}$.

Table 7 Sizing requirements and results at a battery pack specific energy of $400 \mathrm{Wh} / \mathrm{kg}$

\begin{tabular}{lcccc}
\hline & \multicolumn{4}{c}{ UAM vehicle configuration } \\
\cline { 2 - 5 } Vehicle parameter & $\begin{array}{c}\text { Multirotor } \\
\text { (hybrid-electric) }\end{array}$ & $\begin{array}{c}\text { Multirotor } \\
\text { (full-electric) }\end{array}$ & $\begin{array}{c}\text { Tiltrotor } \\
\text { (hybrid-electric) }\end{array}$ & $\begin{array}{c}\text { Tiltrotor } \\
\text { (full-electric) }\end{array}$ \\
\hline Cruise distance, $\mathrm{km}$ & 30 & 30 & 70 & 70 \\
Maximum cruise speed, km/h & 100 & 100 & 270 & 270 \\
Persons on board & 2 & 2 & 4 & 4 \\
Maximum take-off mass, kg & 491 & 620 & 1556 & 1975 \\
Empty mass (excluding energy), kg & 262 & 277 & 1064 & 1154 \\
Energy storage mass, kg & 49 & 163 & 132 & 461 \\
Payload mass, kg & 180 & 180 & 360 & 360 \\
Disk loading, N/m ${ }^{2}$ & 120 & 120 & 400 & 400 \\
Disk area, ${ }^{2}$ & 40.1 & 50.6 & 38.2 & 48.4 \\
Wing loading, N/m ${ }^{2}$ & - & - & 1108 & 1108 \\
Wing span, $\mathrm{m}$ & - & - & 14.4 & 15.0 \\
Wing area, $\mathrm{m}^{2}$ & - & - & 13.8 & 17.5 \\
Wing aspect ratio & - & - & 15 & 15 \\
Best range speed, km/h & 84 & 85 & 170 & 176 \\
Equivalent lift-to-drag ratio & 3.8 & 3.8 & 14.6 & 14.6 \\
Hover C-rate, $1 / \mathrm{h}$ & 1.0 & 1.1 & 2.4 & 2.5 \\
Cruise C-rate, $1 / \mathrm{h}$ & 0.6 & 0.7 & 0.4 & 0.4 \\
Charging C-Rate, $1 / \mathrm{h}$ & 2.4 & 2.4 & 2.4 & 2.4 \\
Charger power required, $\mathrm{kW}$ & 48 & 174 & 132 & 492 \\
\hline
\end{tabular}

The MTOM of all converged UAM vehicles is provided in Fig. 18, where the impact of different battery technology levels can be seen for both powertrain architectures, i.e. hybrid- and full-electric. Generally, it can be noted that all aircraft converge at an MTOM of less than $3175 \mathrm{~kg}$, which is a certification requirement for small category VTOL vehicles declared by the European Union Aviation Safety Agency (EASA) [31]. Thus, all UAM vehicles are primarily feasible with respect to certification and operation. Furthermore, it can be seen that similar trends are propagating through from subsystem to system of interest level. Accordingly, the MTOM reduces quite significantly in the case of full-electric powertrains, which is a result of the advancing battery technology, of course.

For example, the MTOM of the full-electric tiltrotor first reduces by $22 \%$ (from $250 \mathrm{Wh} / \mathrm{kg}$ to $400 \mathrm{Wh} / \mathrm{kg}$ ), and further by $16 \%$ (from $400 \mathrm{Wh} / \mathrm{kg}$ to $550 \mathrm{Wh} / \mathrm{kg}$ ), even though the input parameters for the sizing remain constant and are not adjusted. Therefore, the aircraft design results with regard to MTOM are less sensitive after a battery pack specific energy of $400 \mathrm{Wh} / \mathrm{kg}$ is realized, which is a known trend for electric UAM vehicles.

As previously described in the subsystem results (see Section IV.A), the hybrid-electric UAM vehicles are less sensitive regarding the decrease of MTOM while improving the battery technology, because of the comparably low supplied power ratio. This means that more energy is provided by the kerosene instead of the battery. 


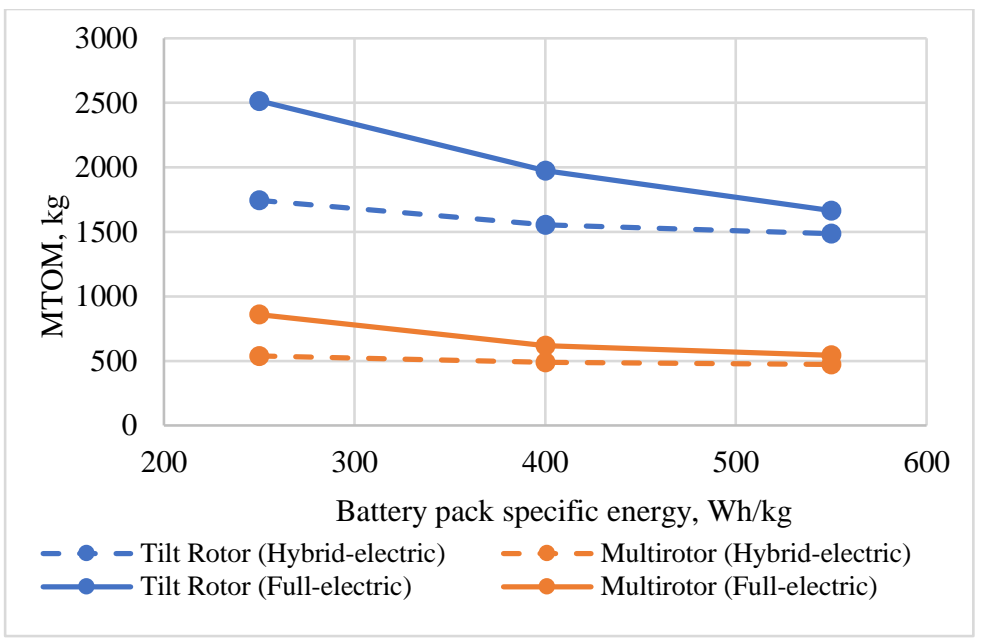

Fig. 18 Maximum take-off mass for both eVTOL configurations and powertrain architectures for different battery technology levels

To provide more insights on the sizing results, several mass fractions are shown in Fig. 19 for the tiltrotor configuration and both powertrain architectures. Here, the impact of advancing battery technology can be found for the hybrid- as well as the full-electric powertrain architecture. Again, the hybrid-electric tiltrotor is less sensitive to advancing battery technology levels compared to the full-electric aircraft. One must be aware that the empty mass fraction does only account for airframe and powertrain components, thus the energy mass is excluded. Accordingly, the energy mass fraction accounts for the battery and kerosene mass, if any. Moreover, the shown payload mass fraction actually does not distinguish between a pilot and a paying passenger. The full design payload of $360 \mathrm{~kg}$ is taken in this case, respectively.

Generally, the energy mass fraction reduces as the battery pack specific energy increases. Again, this is a result of the decreasing MTOM, which reduces the overall energy consumption of the vehicle. As a consequence of the reducing energy mass fraction, the empty mass fraction does not remain constant, but rather increases, whereas the respective gradient flattens between $400 \mathrm{Wh} / \mathrm{kg}$ and $500 \mathrm{Wh} / \mathrm{kg}$. This means that beyond a battery pack specific energy of $400 \mathrm{Wh} / \mathrm{kg}$ aircraft designers may shift their main focus from energy to airframe and propulsion weight again. However, since aircraft design is a multidisciplinary process, all corresponding disciplines must be addressed in order to achieve a high payload mass fraction. Following the described trends, the payload mass fraction constantly increases with advancing battery technology. At $550 \mathrm{Wh} / \mathrm{kg}$ the payload mass fraction of the full-electric tiltrotor is only slightly smaller compared to tiltrotor with the hybrid-electric powertrain. Also, the energy mass fraction falls below the payload mass fraction at this battery pack specific energy. 


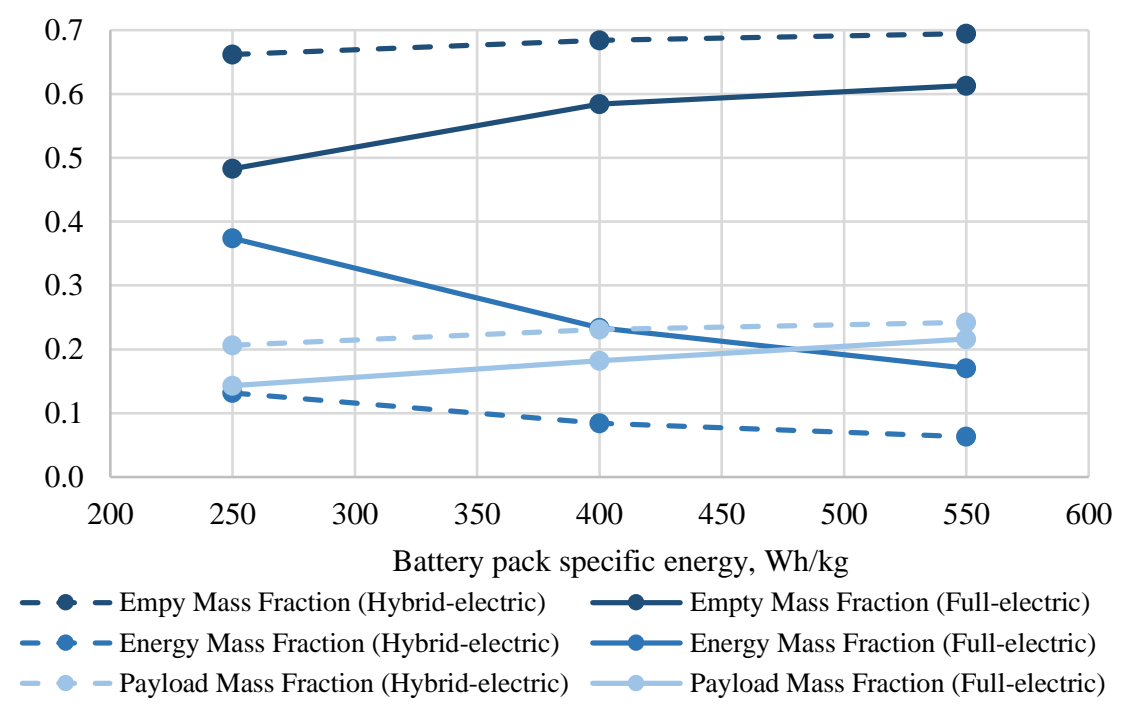

Fig. 19 Mass fractions for the tiltrotor configuration and both powertrain architectures for different battery technology levels

A breakdown of the airframe mass can be found for both tiltrotor configuration architectures, i.e. hybrid- and fullelectric. Generally, the wing and the fuselage are estimated as the highest structural masses compared to the landing gear and the empennage. As before, the trend of decreasing airframe mass can be seen for the underlying battery technology advancements. However, the wing mass of the full-electric tiltrotor is not reduced throughout the sensitivity study. Since there is no weight optimization involved in the current sizing loop, the wing of the tiltrotor is always sized at a constant wing loading, thus no clear trend can be found in this case. As the remaining airframe component masses are mostly estimated with respect to MTOM, the basic trend continues.
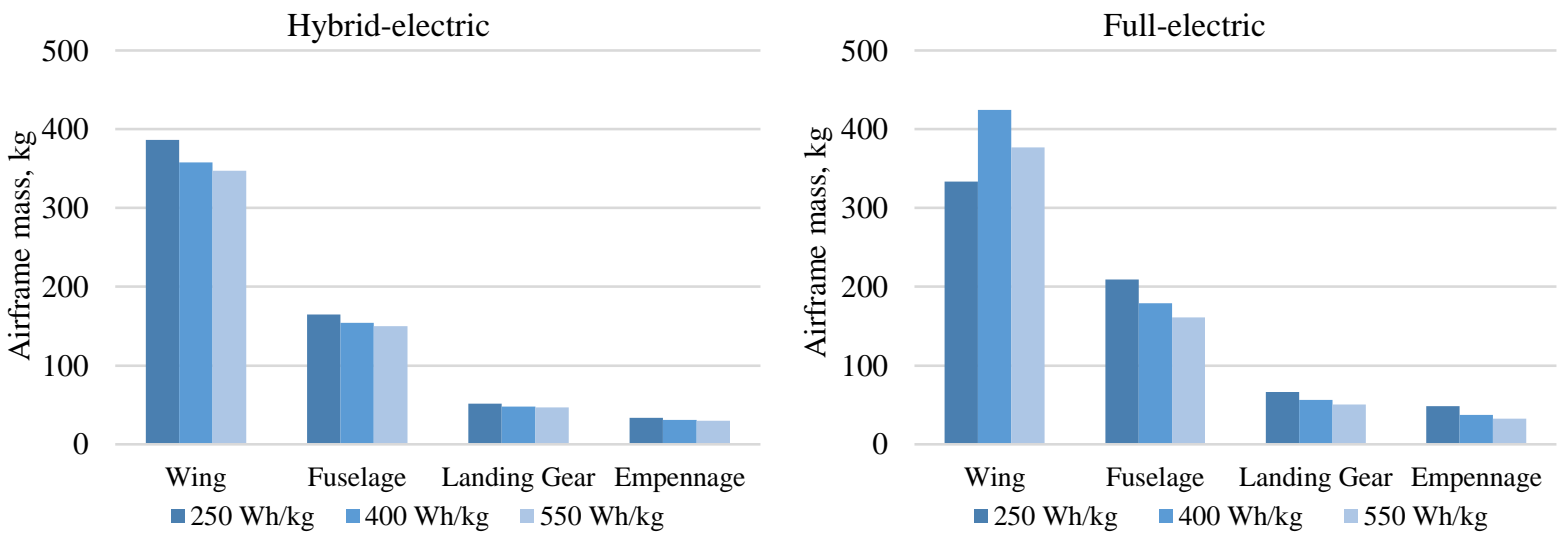

Fig. 20 Airframe mass breakdown for the tiltrotor configuration and both powertrain architectures for different battery technology levels

Regarding the resulting mass fractions in the case of the multirotor configuration in Fig. 22, the general trends remain similar to the tiltrotor configuration as discussed before. Generally, the empty mass fraction of this UAM vehicle configuration is found to be smaller, of course, since no wing and empennage are required. With regard to the underlying sizing mission, a relatively high payload mass fraction can be achieved throughout all the considered battery technology levels. At $550 \mathrm{Wh} / \mathrm{kg}$ payload mass fractions of almost 0.4 for the hybrid-electric and almost 0.35 for the full-electric multirotor can be reached. The energy mass fraction falls well below the payload mass fraction for both powertrain architectures at this battery pack specific energy. 


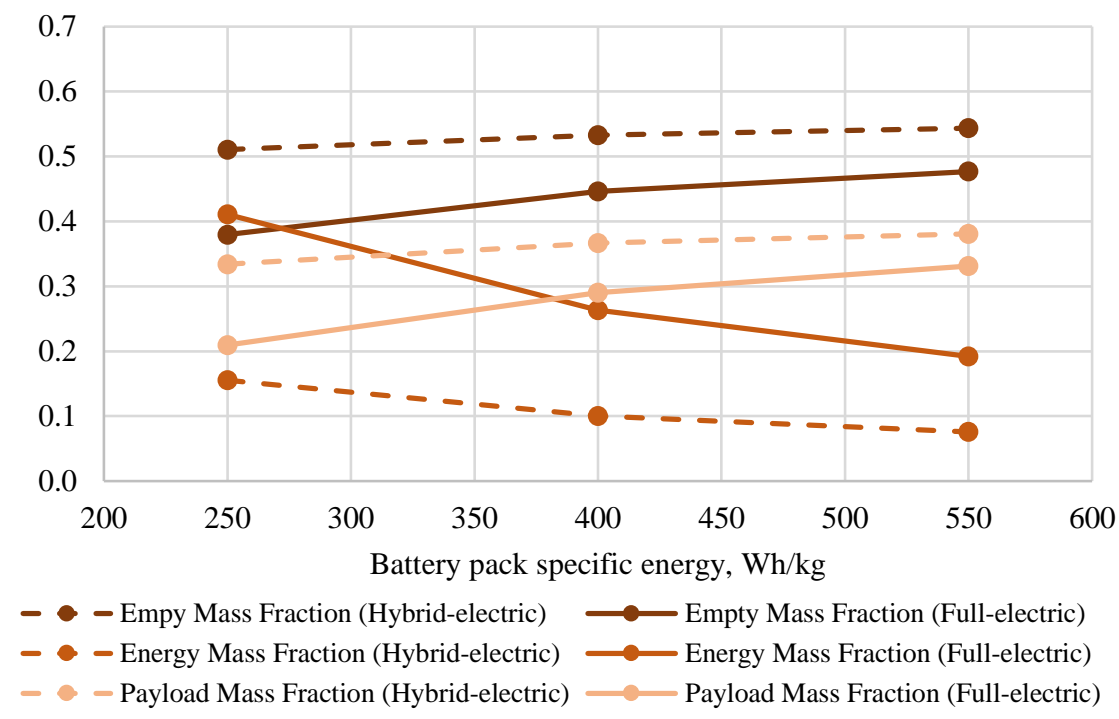

\section{Fig. 21 Mass fractions for the multirotor configuration and both powertrain architectures for different battery technology levels}

The power curves, i.e. propulsive and battery power over the mission time, provide insights into the efficiencies of hybrid- and full-electric eVTOL vehicles. Therefore, both power curves of the resulting tiltrotor at a battery specific energy of $400 \mathrm{Wh} / \mathrm{kg}$ can be found in Fig. 22. Generally, the effect of headwind can be seen as the mission time of the forward flight segments, i.e. cruise climb and cruise, clearly increases compared to no wind conditions.

Regarding the propulsive power, the results are as expected. Here, the power demand peaks in the vertical flight segments, whereas the cruise power is only a minor fraction of it. In some cases, the battery of the tiltrotor is sized by the peak power demand as a result of the maximum C-rate of $2.5 \mathrm{C}$ in this study, which was described in Section II.B. Accordingly, the battery is oversized with regard to the battery specific energy. Thus, longer cruise distances become feasible. However, if longer cruise distances are not required, the disk loading of the tiltrotor must be reduced further. As a consequence, the power demand in hover flight may be reduced.

Comparing the hybrid- and full-electric tiltrotors directly, it can be found that the propulsive power demand of the tiltrotor with full-electric powertrain architecture is higher throughout the entire sizing mission, which is a result of its higher MTOM compared to the hybrid-electric tiltrotor. However, the required power and, by extension, the energy coming from either kerosene and battery or only from battery is higher in the case of the hybrid-electric tiltrotor. The reason for this finding is the lower powertrain efficiency, since the hybrid-electric vehicle uses a gas turbine, and thus the overall efficiency is reduced compared to a full-electric powertrain (see Section II.B). The sensitivities of the just described behavior with regard to different battery pack specific energies will be shown later by the SoS results in Section IV.C.
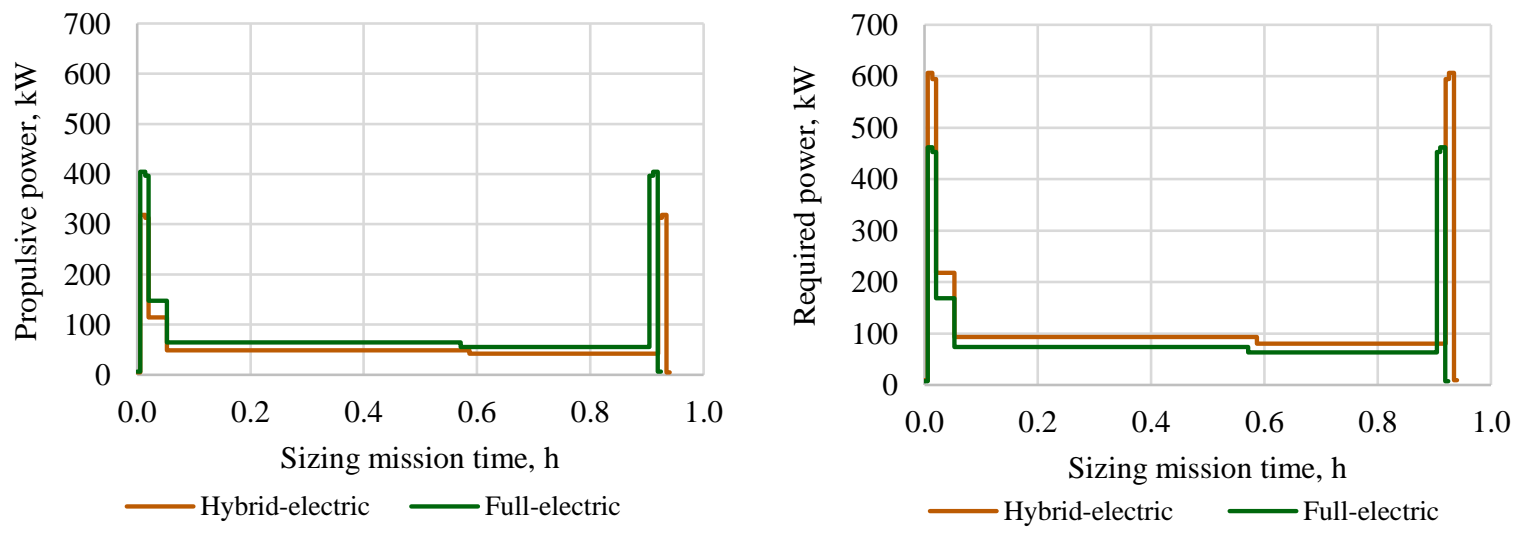

Fig. 22 Power curves of the resulting tiltrotor at a battery pack specific energy of $400 \mathrm{Wh} / \mathrm{kg}$ 
Additionally, the power curves of the resulting multirotor are presented Fig. 23, where a battery pack specific energy of $400 \mathrm{Wh} / \mathrm{kg}$ is considered again. Here, the difference of power demand in hover and cruising flight is less pronounced compared to the tiltrotor (see Fig. 22). This is due to the lower disk loading, which was initially assumed for the multirotor (see Section II.A). However, the cruise power demand is relatively high, since a comparably small equivalent lift-to-drag ratio is achieved for this UAM vehicle configuration. It should also be noted that the sizing mission of the multirotor has a longer duration compared to the tiltrotor, even though the required cruise distance is shorter. This is a consequence of the comparably low true air speed of the multirotor, which leads to an even lower ground speed for the considered headwind conditions (see Section II.A). Apart from that, the results for the multirotor are qualitatively similar to the tiltrotor.
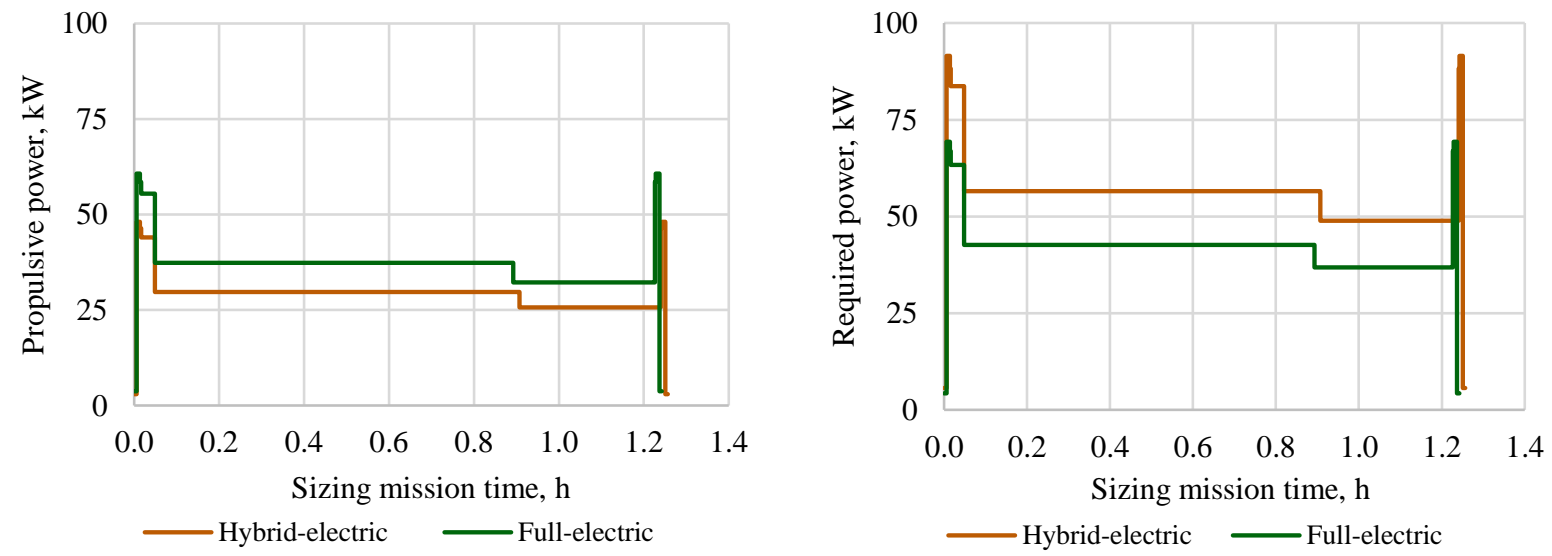

Fig. 23 Power curves of the resulting multirotor at a battery pack specific energy of $400 \mathrm{Wh} / \mathrm{kg}$

\section{Fleet (System of Systems) Results}

1. What is The Impact of Autonomy on the Required Fleet Size for Multirotor in urban Hamburg use case?

\begin{tabular}{|l|l|l|l|l|l|}
\hline Use Case & Vehicle & Powertrain & Capacity & Specific Energy & Operations \\
\hline Hamburg: Urban & Multirotor & Electric & 2 POB & $250 \mathrm{Wh} / \mathrm{kg}$ & Piloted vs Autonomous \\
\hline
\end{tabular}

The Fig. 24 below shows the fleet size variation and the effect on measures of effectiveness of UAM SoS. For the Autonomous fleet 48 Aircraft could successfully cater $90 \%$ of missions within 15 -minute time limit. For Piloted fleet, 84 Aircraft are needed to cater $90 \%$ successful missions. This is due to the fact that, the autonomous vehicles can carry up to 2 passengers on board where as non-autonomous can only carry 1 passenger apart from pilot.

Dead head effects due to fleet can also be visualized. Deadheading is the necessary evil in the UAM SoS, when there is a fleet vs passenger flight demand mismatch at the vertiports, the simulation reallocates the aircraft based on the demand for the flight $\&$ the availability of aircraft, often when the fleet size is small, the aircraft is reallocated to adjust the demand-supply issue. Thus, the reason for success percentage to be low at lower fleet size is also due to fleet organization.

Fleet Total Energy provides the energy required by Autonomous and piloted fleet and it can be seen that, as the fleet size increased there is an energy peak (due to deadhead/ reallocation) and decreases and saturates. At respective "success" fleet sizes, Autonomous fleet require almost $\sim 30 \%$ Less energy than piloted feet

Average wait time (min) is an important metric, within the success or fail criteria, it can be understood how the system is clogged. The Simulation also shares the information about the clogged vertiports, but not presented in the results. 


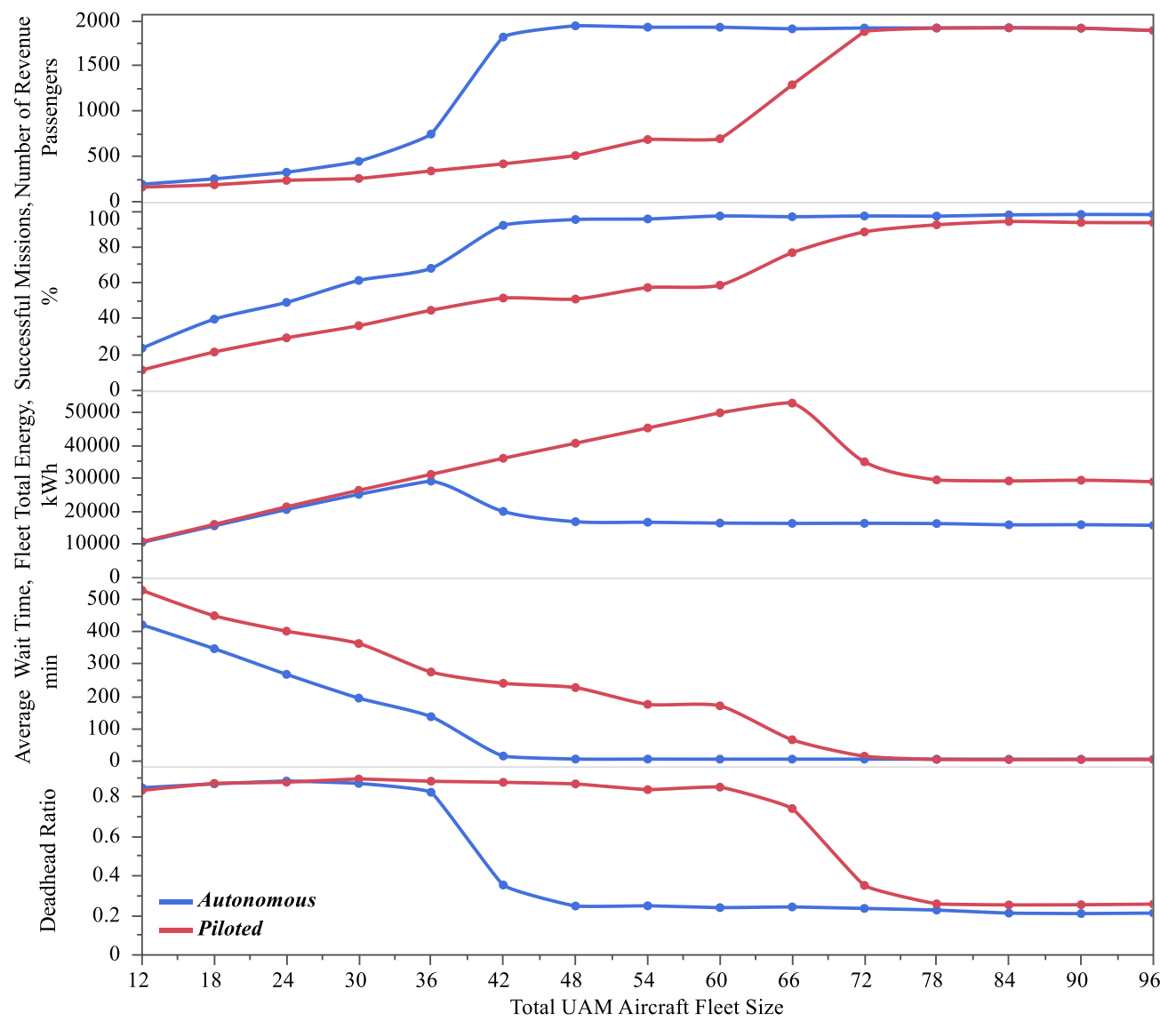

Fig. 24 Multirotor fleet for urban Hamburg operations, average mission radius of $15 \mathrm{~km}$, autonomous vs piloted, full-electric, and battery pack specific energy of $250 \mathrm{Wh} / \mathrm{kg}$

2. What is The Impact of Autonomy on The Required Fleet Size for Tiltrotor in Suburban California Use Case?

\begin{tabular}{|l|l|l|l|l|l|}
\hline Use Case & Vehicle & Powertrain & Capacity & Specific Energy & Operations \\
\hline California: suburban & Tiltrotor & Electric & 4 POB & $250 \mathrm{Wh} / \mathrm{kg}$ & Piloted vs Autonomous \\
\hline
\end{tabular}

As shown in Fig. 25 below, the effect of autonomy is not great as compared to multirotor, the main reason being the maximum number of passengers on board with autonomy for tiltrotor is 4 and multirotor is 2 . With piloted Multirotor will have to dedicate $50 \%$ of load factor to pilot and Tiltrotor have to dedicate only $25 \%$ of its load factor. The fleet required for $90 \%$ success criteria with autonomous operation is 42 and Piloted it is 48 vehicles. The effect of autonomy can we seen in Total Fleet Energy for the fleet sizes, it is about 12\% less than the Piloted fleet. It should be noted that the Tiltrotor fly at higher speed and larger distance than the Multirotor deployed in Urban - Hamburg use case. 


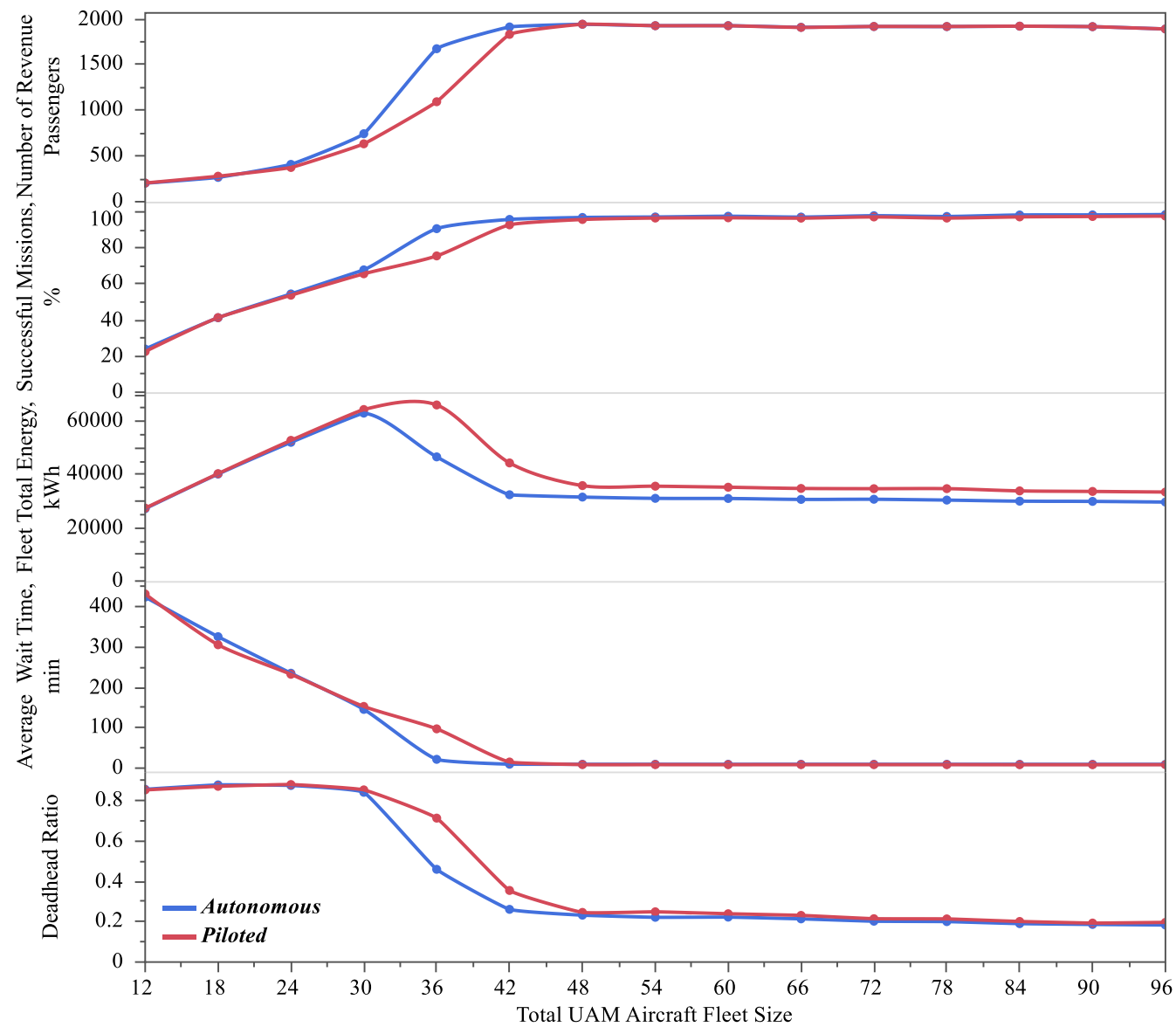

Fig. 25 Tiltrotor fleet for suburban California operations, average mission radius of $35 \mathrm{~km}$, autonomous vs piloted, full-electric, and battery pack specific energy of $250 \mathrm{Wh} / \mathrm{kg}$

The above System of System effect due to change in System of Interest and its Subsystem architecture is interesting to understand the point of inflection where the success percentage or energy consumption has a inflection point and saturates. Further the law of diminishing returns follows. It should be noted that this result is specific to passenger demand assumed, vertiport network and vehicle design \& technology assumptions and finally dispatching CONOPS.

3. What is The Impact of Hybrid-and Full-Electric Powertrain Architectures on The Required Fleet Size for the Multirotor Urban Hamburg Use Case?

\begin{tabular}{|l|l|l|l|l|l|}
\hline Use Case & Vehicle & Powertrain & Capacity & Specific Energy & Operations \\
\hline Hamburg: Urban & Multirotor & Hybrid vs Electric & 2 POB & $250 \mathrm{Wh} / \mathrm{kg}$ & Piloted \\
\hline
\end{tabular}

Fig. 26 below represents the effect of power train subsystem (full-electric or hybrid-electric) on the SoS measures of effectiveness. Only non-autonomous multirotor vehicles are considered in the below plot due to page limitation. The $90 \%$ success criteria for hybrid-electric multirotor is met by fleet size of 84 aircraft and for All electric, the required fleet size is 78 aircraft. The more predominant effect can be seen in the Energy required, hybrids require higher energy. Even for the same fleet size required for $90 \%$ success, the total fleet energy required for hybrid aircraft operating 24 hours is $25 \%$ more than all electric Fleet. 


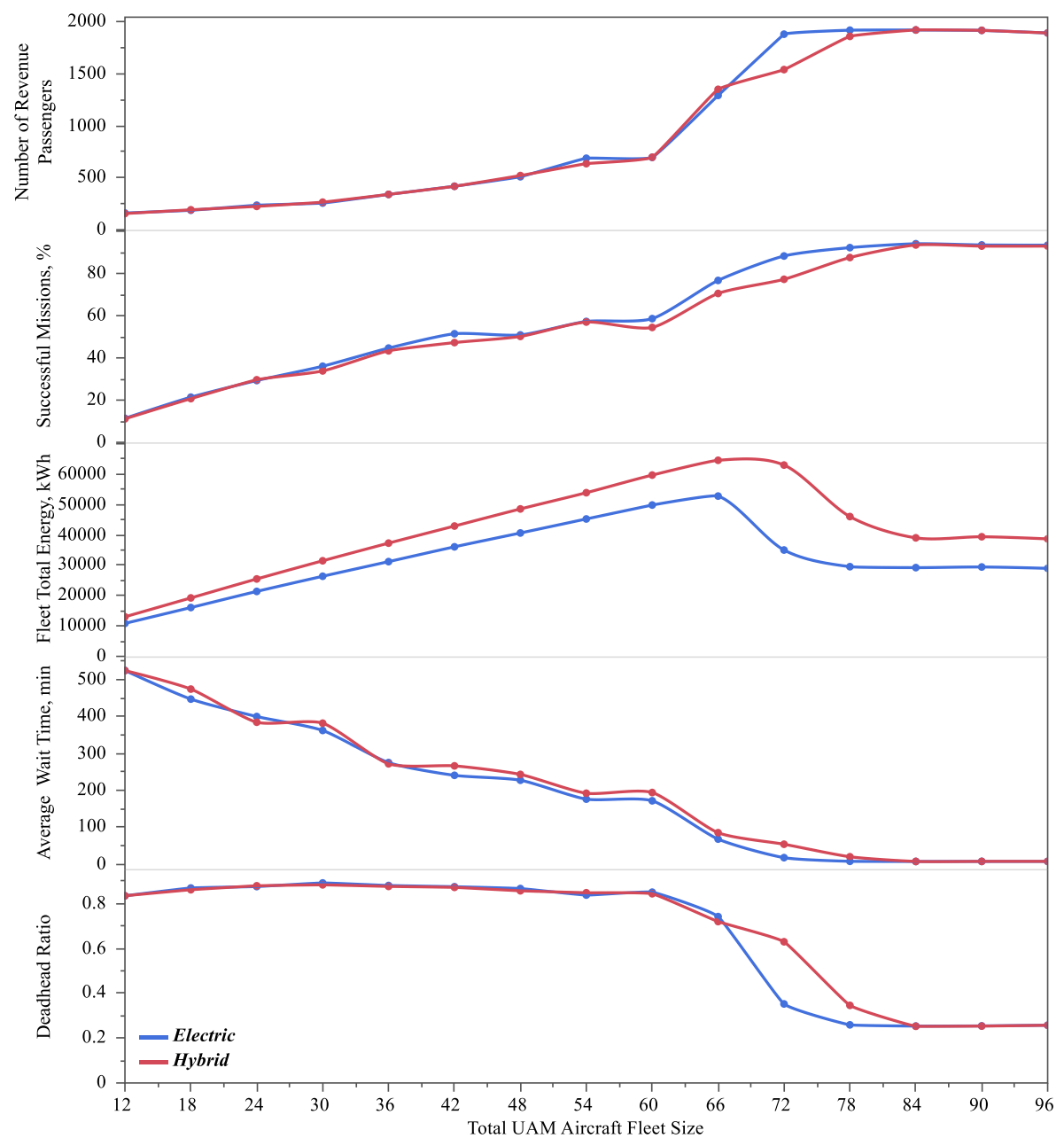

Fig. 26 Multirotor fleet for urban Hamburg operations, piloted, hybrid-electric vs full-electric, average mission distance of $15 \mathrm{~km}$ and battery pack specific energy of $250 \mathrm{Wh} / \mathrm{kg}$

4. What is The Effect of Battery Pack Specific Energy on the Energy Consumed by the Fleet under The Consideration of Hybridization and Autonomy?

\begin{tabular}{|l|l|l|l|l|l|}
\hline Use Case & Vehicle & Powertrain & Capacity & Specific Energy & Operations \\
\hline Hamburg: Urban & $\begin{array}{l}\text { Multirotor \& } \\
\text { Tiltrotor }\end{array}$ & Hybrid vs Electric & 4 POB & $\begin{array}{l}\mathbf{2 5 0} \text { vs 400 vs } \\
\mathbf{5 5 0} \mathbf{~ W h} / \mathbf{k g}\end{array}$ & $\begin{array}{l}\text { Piloted vs } \\
\text { Autonomous }\end{array}$ \\
\hline
\end{tabular}

The results from above Fig. 24, Fig. 25, Fig. 26 showed the fleet size effects for one battery specific energy of $250 \mathrm{Wh} / \mathrm{kg}$. In the below Fig. 27, we can evaluate the battery pack specific energy effect (Subsystem level) on the System of Systems (fleet total energy). The battery effect integrated to multirotor is visualized in Fig. 27 and tiltrotor in Fig. 28.

Few takeaways are:

- Higher the battery specific energy, lesser the total fleet energy. Which is a complex effect considering mass of battery, overall vehicle performance and energy consumption, recharge and dispatching adjustments for supply demand balance.

- The energy peaks and then drops due to complex factors: dispatch adjustments for supply-demand balance, balance between waiting for vehicle load factor to fill and start the mission within 15 minutes to improve success criteria, and recharge time based on energy consumption from previous flight. Due to this with limited fleet size there is more energy consumed due to inefficient load factors, increased dead heading, the other dispatch 
balancing factors. With larger fleet, the inefficiencies fade out and the energy consumption of fleet drops. Then there is law of diminishing returns, where, beyond certain fleet size there is no further improvement in energy.

- The highest Battery specific energy $(550 \mathrm{Wh} / \mathrm{kg})$ when compared to lowest Battery specific energy ( $250 \mathrm{Wh} / \mathrm{kg})$, needs $60 \%$ less total fleet energy for Electric, Piloted case. For Autonomous hybrid fleet, it is just $25 \%$ better.

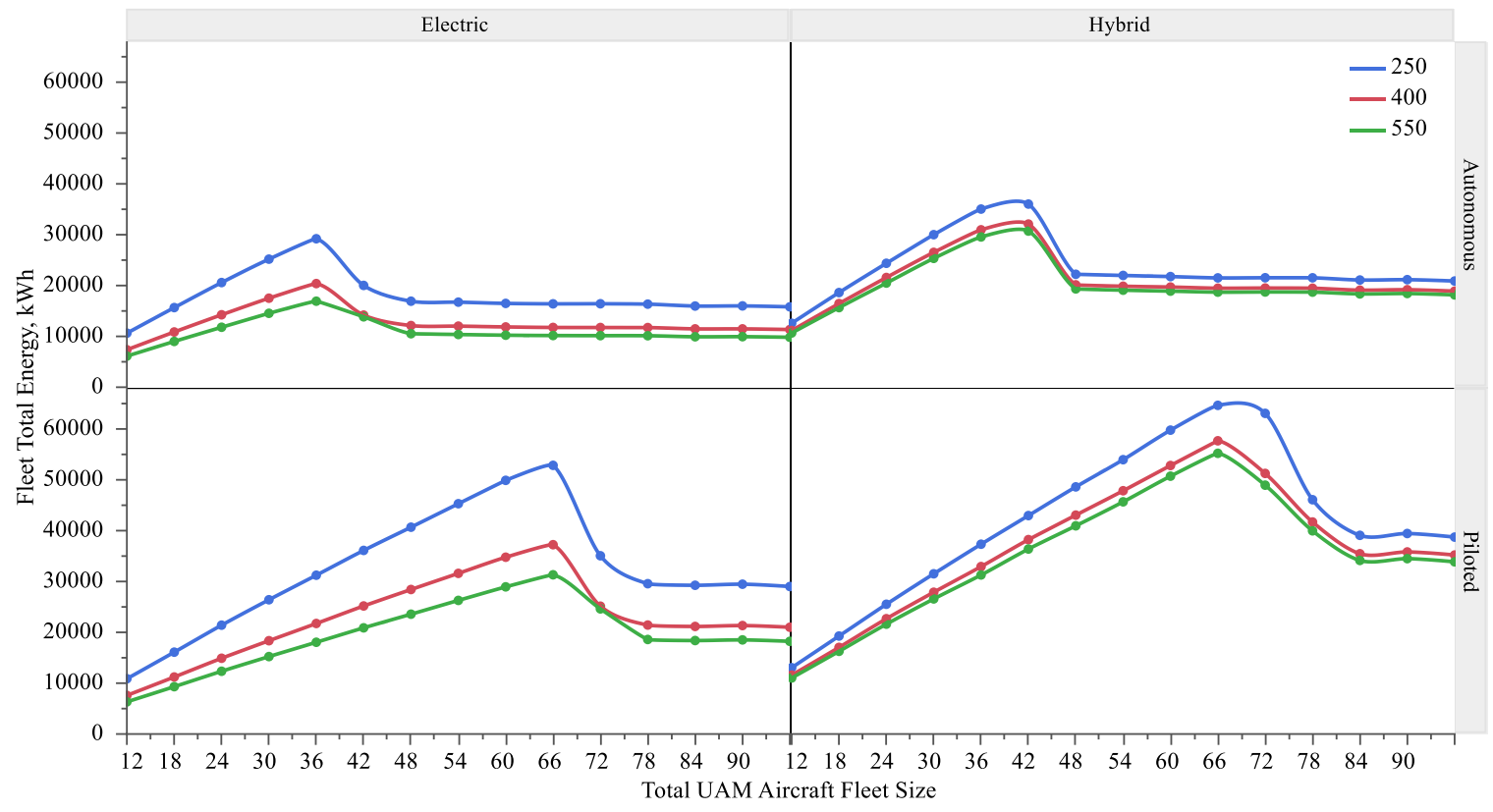

Fig. 27 Multirotor fleet for urban Hamburg operations, average mission radius of $15 \mathrm{~km}$, effect of advancing battery specific energy on fleet total energy for 24-hour operations

Fig. 28 shows the effect of battery specific energy on total fleet energy for a tiltrotor. Note that this is for a larger ( $35 \mathrm{~km}$ avg distance) operation with maximum 4 passengers on board, compared to multirotor operated with maximum 2 passengers on board and smaller urban missions (15 km average distance). 


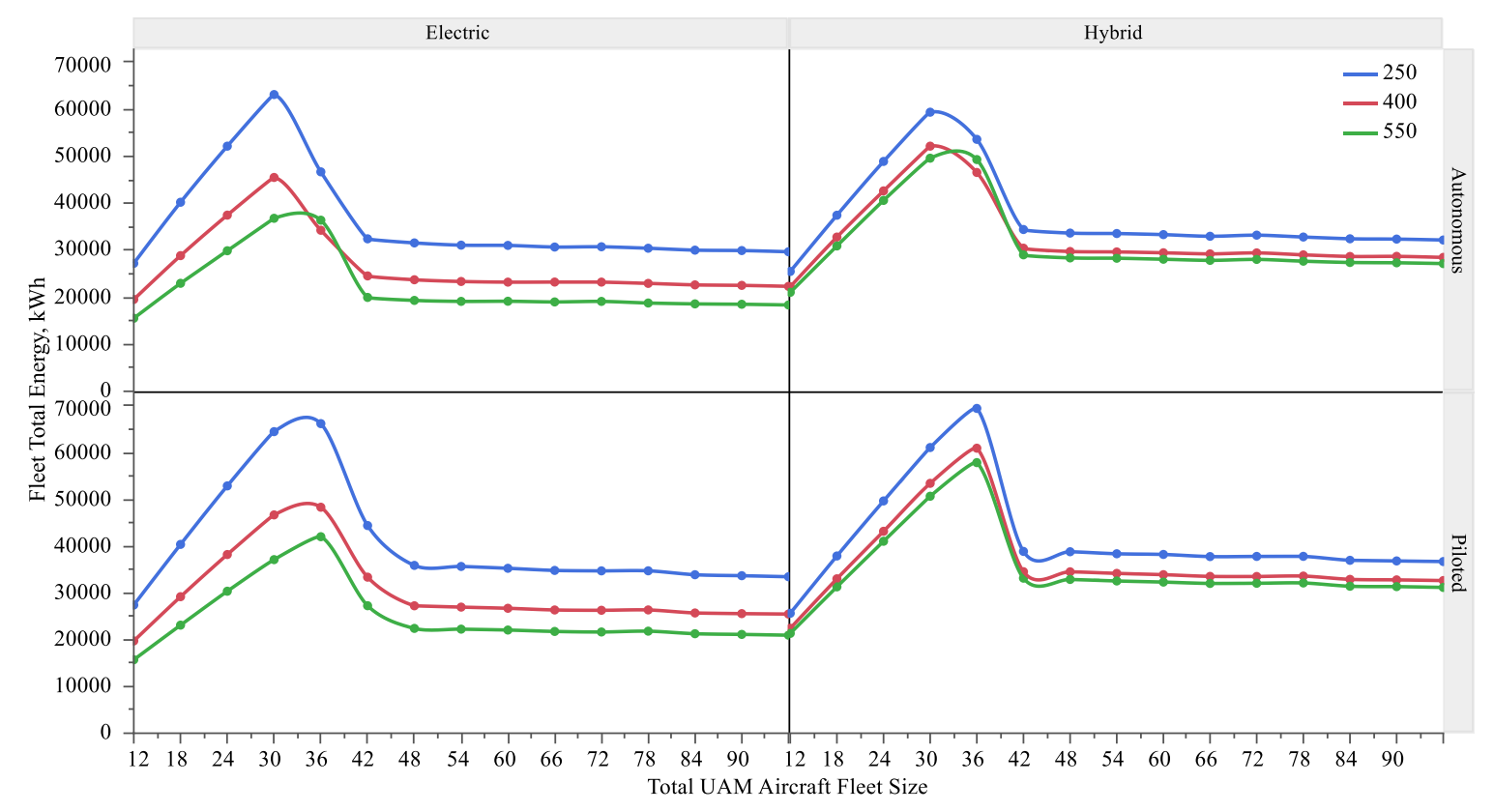

Fig. 28 Tiltrotor fleet for suburban California operations, average mission radius of $35 \mathrm{~km}$, effect of advancing battery specific energy on fleet total energy for 24-hour operations

\section{Conclusion and Future Work}

The research questions addressed shows that the framework can successfully evaluate the multilevel impacts. The subsystem and power train architectures, autonomy and battery impacts were embedded on multirotor and tiltrotor vehicle design evolution. Several homogeneous fleets were formed and evaluated via an Agent-Based Simulation.

The research question 1 demonstrated that a 75\% larger fleet size is required if the UAM is operated with Piloted fleet as compared to autonomous fleet. It should be noted that the successful operation of $90 \%$ of mission within 15 minutes is an assumed evaluation metric. Also, this result changes with different demand distribution, vertiport distances and waiting time cutoff.

The research question 3 addressed the effect of Hybrid Electric and All electric powertrain effect. Even for the same fleet size required for $90 \%$ success, the total fleet energy required for hybrid aircraft operating 24 hours is $25 \%$ more than all electric Fleet. The highest Battery specific energy $(500 \mathrm{Wh} / \mathrm{kg}$ ) when compared to lowest Battery specific energy $(250 \mathrm{Wh} / \mathrm{kg})$, needs $60 \%$ less total fleet energy for Electric, Piloted case. For Autonomous hybrid fleet, it is just $25 \%$ better. Note that these results are specific to technology and performance assumptions, demand, and specific vertiport network.

Also, the complex nonlinear phenomenon of energy requirements vs fleet size required to cater to demand vs fleet distribution vs Waiting time factor vs deadheading can be understood. The sensitivity of different Subsystem-system of interest and system of system parameters can be understood using the SoS UAM framework. It is important to deploy ideal fleet and vehicles for the given demand to make UAM operations efficient and sustainable. The technology investments can be made using this framework based on fleet requirement and sustainability targets.

As shown in Fig. 29 , in future higher fidelity aircraft design, more CONOPS evaluations, operation scenarios and detailed noise optimum trajectories will be analyzed. Furthermore, the vertiport network optimization based on passenger demand for respective urban or suburban use case is important. Life cycle analysis is very much integral part of UAM assessment.

The focus of the research was to attempt to develop a multi-level subsystem-system-system of systems assessment framework and through sensitivity study, it was proven to be successful. Improved demand data models will be used in future studies. 


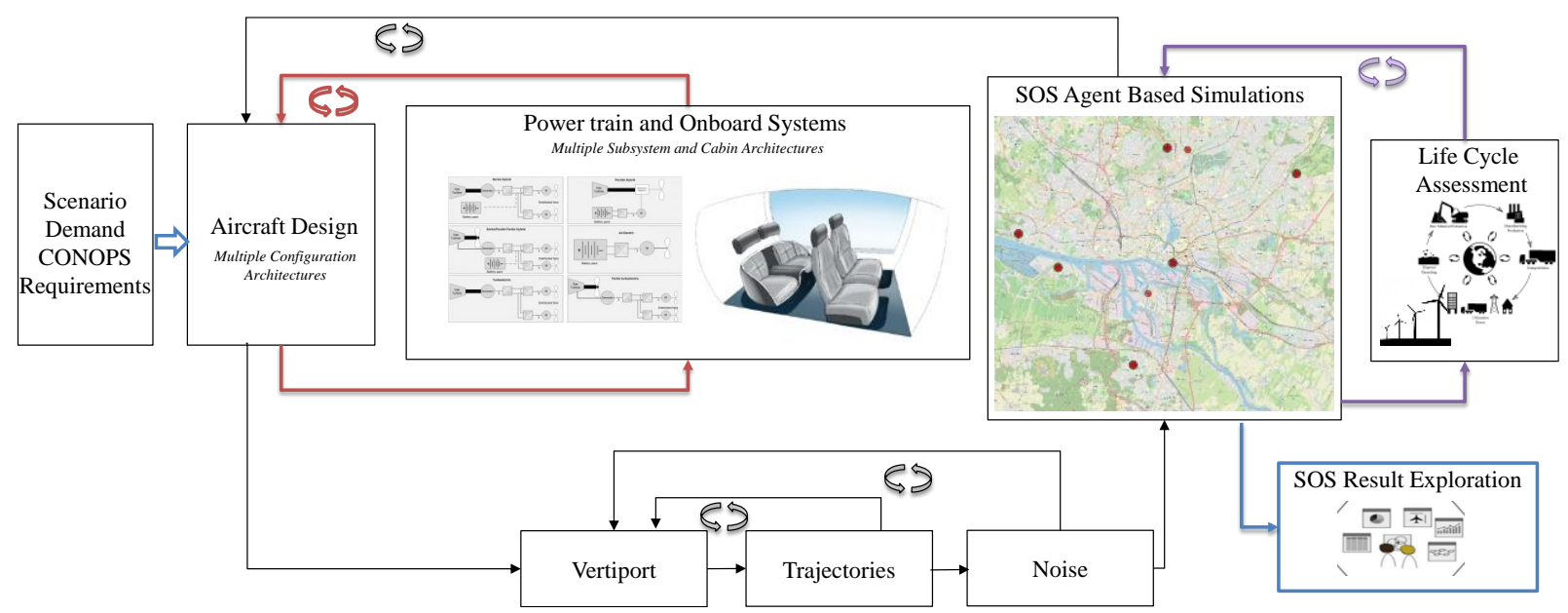

Fig. 29 System of System UAM collaborative framework

\section{References}

[1] J. Holden, N. Goel, Fast-Forwarding to the Future of On-Demand, Urban Air Transportation, 2016. URL https://www.uber.com/elevate.pdf.

[2] R. McDonald, B. German, eVTOL Stored Energy Overview, in: Uber Elevate Summit, 2017, pp. 14-20.

[3] EHang, EHang 216. URL https://www.ehang.com/ehangaav/

[4] Volocopter, Volocity. URL https://www.volocopter.com/de/product/

[5] Jaunt Air Mobility, Jaunt Journey Air Taxi. URL https://jauntairmobility.com/aircraft.html

[6] Wisk, Cora by Wisk. URL https://wisk.aero/cora/

[7] Joby Aviation, Joby S4. URL https://www.jobyaviation.com/

[8] Lilium, Lilium Jet. URL https://lilium.com/the-jet

[9] ISO/IEC/IEEE 21839:2019, Systems and software engineering - system of systems (sos) considerations in life cycle stages of a system, ISO/IEC/IEEE International Standard.

[10] H. Farhangi, D. Konur, System of Systems Architecting Problems: Definitions, Formulations, and Analysis, Procedia Computer Science 140 (2018) 29-36. doi: https://doi.org/10.1016/j.procs.2018.10.289.

[11] M. W. Maier, Architecting principles for systems-of-systems, Systems Engineering 1 (4) (1998) 267-284. doi:https://doi.org/10.1002/(SICI) 1520-6858(1998)1:4<267::AID-SYS3>3.0.CO;2-D.

[12] J. S. Dahmann, K. J. Baldwin, Understanding the Current State of US Defense Systems of Systems and the Implications for Systems Engineering, in: 2008 2nd Annual IEEE Systems Conference, 2008, pp. 1-7.

[13] C. B. Nielsen, P. G. Larsen, J. Fitzgerald, J. Woodcock, J. Peleska, Systems of systems engineering: Basic concepts, model-based techniques, and research directions, ACM Computing Surveys 48 (2) (Sep. 2015). doi: $10.1145 / 2794381$.

[14] W. Johnson, C. Silva, E. Solis, Concept Vehicles for VTOL Air Taxi Operations, in: AHS Technical Conference on Aeromechanics Design for Transformative Vertical Flight, 2018.

[15] A. R. Kadhiresan, M. J. Duffy, Conceptual Design and Mission Analysis for eVTOL Urban Air Mobility Flight Vehicle Configurations, in: AIAA Aviation 2019 Forum, 2019. doi:10.2514/6.2019-2873.

[16] A. Brown, W. L. Harris, A Vehicle Design and Optimization Model for OnDemand Aviation, in: AIAA SciTech 2018 Forum, Structures, Structural Dynamics, and Materials Conference, 2018. doi:10.2514/6.2018-0105.

[17] N. Andre, M. Hajek, Robust Environmental Life Cycle Assessment of Electric' VTOL Concepts for Urban Air Mobility, in: AIAA Aviation 2019 Forum, 2019. doi:10.2514/6.2019-3473.

[18] M. Clarke, J. Smart, E. M. Botero, W. Maier and J. J. Alonso, Strategies for Posing a Well-Defined Problem for Urban Air Mobility Vehicles, in: AIAA Scitech 2019 Forum, 2019. doi:10.2514/6.2019-0818. 
[19] K. O. Ploetner, C. Al Haddad, C. Antoniou, F. Frank, M. Fu, S. Kabel, C. Llorca, R. Moeckel, A. T. Moreno, A. Pukhova, R. Rothfeld, M. Shamiyeh, A. Straubinger, H. Wagner, Q. Zhang, Long-term application potential of urban air mobility complementing public transport: an upper bavaria example, CEAS Aeronautical Journal 11 (4) (2020) 991-1007. doi:10.1007/s13272-020-00468-5.

[20] M. Husemann, E. Stumpf, N. Dirks, G. Walther, Towards the Design of CostEfficient Urban Air Taxi Systems, in: AIAA Scitech 2021 Forum. doi:10.2514/ 6.2021-1515.

[21] L. W. Kohlman, M. D. Patterson, System-Level Urban Air Mobility Transportation Modeling and Determination of Energy-Related Constraints, in: AIAA Aviation 2018 Forum, Aviation Technology, Integration, and Operations Conference, 2018. doi:10.2514/6.2018-3677.

[22] Ciampa, P. D., Prakasha, P. S., Torrigiani, F., Walther, J.-N., Lefebvre, T., Bartoli, N., Timmermans, H. et al. "Streamlining Cross-Organizational Aircraft Development: Results from the AGILE Project," AIAA Aviation 2019 Forum, 2019 p. 3454. DOI: 10.2514/6.2019-3454.

[23] Prakasha, P. S., Boggero, L., Fioriti, M., Aigner, B., Ciampa, P. D., Anisimov, K., Isianov, A., "Collaborative system of systems multidisciplinary design optimization for civil aircraft: AGILE EU project," 18th AIAA/ISSMO Multidisciplinary Analysis and Optimization Conference, p. 4142, 2017.

[24] Fioriti, M, Boggero, L., Corpino, S., Prakasha, P. S., Ciampa, P. D., Nagel, B. "The effect of sub-systems design parameters on preliminary aircraft design in a multidisciplinary design environment," Transportation Research Procedia 29, pp. 135-145, 2018.

[25] Prakasha, P. S., Nam, T., Perullo, C. and Mavris, D. N., "Non Deterministic Approach for Advanced Aircraft Configuration Design under Uncertainty," $15^{\text {th }}$ AIAA/ISSMO Multidisciplinary Analysis and Optimization Conference, p. 2182, 2014.

[26] Asmer, L., Pak, H., Prakasha, P. S., Schuchardt, B. I., Weiand, P., Meller, F., Torens, C., Becker, D., Zhu, C., Schweiger, K., and Volkert, A., "Urban Air Mobility Use Cases, Missions and Technology Scenarios for the HorizonUAM Project," AIAA Aviation 2021 Forum, 2021.

[27] Prakasha, P., Ratei, P., Naeem, N., Nagel, B., Papantoni, V., Villacis, S., Brand, U., Vogt, T., "Urban Air Mobility Vehicle and Fleet-level Life-Cycle Assessment Using a System-of-Systems Approach," AIAA Aviation 2021, American Institute of Aeronautics and Astronautics, 2021.

[28] C. Silva, W. R. Johnson, E. Solis, M. D. Patterson, K. R. Antcliff, VTOL Urban Air Mobility Concept Vehicles for Technology Development, AIAA Aviation 2018 Forum, Aviation Technology, Integration, and Operations Conference, 2018. .DOI:10.2514/6.2018-3847.

[29] De Vries, R., Brown, M., Vos, R., "Preliminary sizing method for hybrid-electric distributed-propulsion aircraft," Journal of Aircraft, 2019, 56:6, pp. 2172-2188.

[30] Vegh, J. Michael, et al, "Current capabilities and challenges of ndarc and suave for evtol aircraft design and analysis," 2019 AIAA/IEEE Electric Aircraft Technologies Symposium (EATS). IEEE, 2019. pp. 1-19.

[31] European Union Aviation Safety Agency, Special Condition: Vertical Take-Off and Landing (VTOL) Aircraft.

[32] Kilkis, S., Naeem, N., Prakasha, P. S., Nagel, B., "A Python Modelling and Simulation Toolkit for Rapid Development of System of Systems Inverse Design (SoSID) Case Studies," AIAA Aviation 2021 Forum, 2021.

[33] P. D. Vascik, Systems Analysis of Urban Air Mobility Operational Scaling, Ph.D. thesis, Massachusetts Institute of Technology, MIT International Center for Air Transportation. 